\title{
Springer correspondence for the split symmetric pair in type A
}

\author{
Chen, Tsao-Hsien
}

2018-11

Chen , T-H , Vilonen , K \& Xue , T 2018 , ' Springer correspondence for the split symmetric pair in type A ' , Compositio Mathematica , vol. 154 , no. 11 , pp. 2403-2425 . https://doi.org/10.1112/S0010437X180

http://hdl.handle.net/10138/307127

https://doi.org/10.1112/S0010437X18007443

cc_by

acceptedVersion

Downloaded from Helda, University of Helsinki institutional repository.

This is an electronic reprint of the original article.

This reprint may differ from the original in pagination and typographic detail.

Please cite the original version. 


\title{
SPRINGER CORRESPONDENCE FOR THE SPLIT SYMMETRIC PAIR IN TYPE $A$
}

\author{
TSAO-HSIEN CHEN, KARI VILONEN, AND TING XUE
}

\begin{abstract}
In this paper we establish Springer correspondence for the symmetric pair $(\mathrm{SL}(N), \mathrm{SO}(N))$ using Fourier transform, parabolic induction functor, and a nearby cycle sheaves construction due to Grinberg. As applications, we obtain results on cohomology of Hessenberg varieties and geometric constructions of irreducible representations of Hecke algebras of symmetric groups at $q=-1$.
\end{abstract}

\section{INTRODUCTION}

In CVX1] we have initiated a study of Springer correspondence for symmetric spaces, in particular, in the split case of type $A$. There we compute Fourier transforms of IC sheaves supported on certain nilpotent orbits using resolutions of singularities of nilpotent orbit closures. In this paper we study the problem in general in the split case of type $A$ replacing the resolutions with a nearby cycle sheaves construction due to Grinberg [G1, G2. We have obtained partial results in CVX1, CVX2.

Let us call an irreducible IC sheaf supported on a nilpotent orbit a nilpotent orbital complex. We show that the Fourier transform gives a bijection between nilpotent orbital complexes and certain representations of (extended) braid groups. We identify these representations of (extended) braid groups and construct them explicitly in terms of irreducible representations of Hecke algebras of symmetric groups at $q=-1$. This bijection can be viewed as Springer correspondence for the symmetric pair $(\mathrm{SL}(N), \mathrm{SO}(N))$. Let us note that the fact that representations of (affine) Hecke algebras at $q=-1$ arise in this situation was already observed by Grojnowski in his thesis $\mathrm{Gr}$.

The proof of our main result, Theorem 4.1, makes use of a nearby cycle sheaves construction due to Grinberg [G1, G2] and smallness property of maps associated to certain $\theta$-stable parabolic subgroups. In more details, Grinberg's nearby cycle sheaves and their twisted version produce IC sheaves whose Fourier transforms are supported on the nilpotent cone. Those IC sheaves behave like "cuspidal sheaves" in the sense that they do not appear as direct summands of parabolic inductions. On the other hand, the smallness property

Tsao-Hsien Chen was supported in part by the AMS-Simons travel grant.

Kari Vilonen was supported in part by NSF grants DMS-1402928 \& DMS-1069316, the Academy of Finland, the ARC grant DP150103525, the Humboldt Foundation, and the Simons Foundation.

Ting Xue was supported in part by the ARC grants DE160100975, DP150103525 and the Academy of Finland. 
mentioned above implies a simple description of the images of parabolic induction functors (Proposition 3.1, Proposition [3.2). Those results together with a counting lemma (Lemma 4.2) imply Theorem 4.1. As corollaries, we obtain criteria for nilpotent orbital complexes to have full support Fourier transforms (Corollary 4.8, Corollary 4.9) and results on cohomology of Hessenberg varieties (Theorem 5.1).

Our method appears to be applicable to general symmetric pairs or polar representations studied in G2 and we hope to return to this in future work.

Let us mention that in [LY], the authors show that one can obtain all nilpotent orbital complexes using spiral induction functors introduced in [LY] (in fact, they consider more general cyclically graded Lie algebras settings). Using their results and Theorem 4.1, we show that all irreducible representations of Hecke algebras of symmetric groups at $q=-1$ appear in the intersection cohomology of of Hessenberg varieties, with coefficient in certain local systems (see Theorem 6.1). This gives geometric constructions of irreducible representations of Hecke algebras of symmetric groups at $q=-1$ and provides them with a Hodge structure.

The paper is organized as follows. In Section 2 we recall some facts about symmetric pairs and introduce a class of representations of equivariant fundamental groups. In Section 3 we study parabolic induction functors for certain $\theta$-stable parabolic subgroups. In Section 4 , we prove Theorem 4.1, the Fourier transform defines a bijection between the set of nilpotent orbital complexes and the class of representations of equivariant fundamental groups introduced in Section 2, In Section 5 and Section [6, we discuss applications of our results to cohomology of Hessenberg varieties and representations of Hecke algebras of symmetric groups at $q=-1$. Finally, in Section 7 , we propose a conjecture that gives a more precise description of the bijection in Theorem 4.1.

Acknowledgements. We thank Misha Grinberg for helpful conversations and for providing a proof of Theorem 4.4; an extension of his earlier work in [G2]. TC thanks ChengChiang Tsai for useful discussions and thanks the Institute of Mathematics Academia Sinica in Taipei for support, hospitality, and a nice research environment. KV and TX thank the Research Institute for Mathematical Sciences in Kyoto for support, hospitality, and a nice research environment.

\section{PRELiminaries}

2.1. Notations. For $e \geq 2$, a partition $\lambda$ of a positive integer $k$ is called $e$-regular if the multiplicity of any part of $\lambda$ is less than e. In particular, a partition is 2-regular if and only if it has distinct parts. Let us denote by $\mathcal{P}(k)$ the set of all partitions of $k$ and by $\mathcal{P}_{2}(k)$ the set of all 2-regular partitions of $k$.

We denote by $\mathcal{H}_{k,-1}$ the Hecke algebra of the symmetric group $S_{k}$ with parameter -1 . More precisely, $\mathcal{H}_{k,-1}$ is the $\mathbb{C}$-algebra generated by $T_{i}, i=1, \ldots, k-1$, with the following 
relations

$$
\begin{gathered}
T_{i} T_{j}=T_{j} T_{i} \text { if }|i-j| \geq 2, i, j \in[1, k-1], T_{i} T_{i+1} T_{i}=T_{i+1} T_{i} T_{i+1}, i \in[1, k-2], \\
T_{i}^{2}=q+(q-1) T_{i}, \text { where } q=-1, i \in[1, k-1] .
\end{gathered}
$$

It is shown in [DJ] that the set of isomorphism classes of irreducible representations of $\mathcal{H}_{k,-1}$ is parametrized by $\mathcal{P}_{2}(k)$. For $\mu \in \mathcal{P}_{2}(k)$, we write $D_{\mu}$ for the irreducible representation of $\mathcal{H}_{k,-1}$ corresponding to $\mu$.

For a real number $a$, we write $[a]$ for its integer part.

2.2. The split symmetric pair $(\mathrm{SL}(N), \mathrm{SO}(N))$. Let $G=\mathrm{SL}(N)$ and $\theta: G \rightarrow G$ an involution such that $K=G^{\theta}=\mathrm{SO}(N)$ and write $\mathfrak{g}=$ Lie $G$. We have $\mathfrak{g}=\mathfrak{g}_{0} \oplus \mathfrak{g}_{1}$, where $\left.\theta\right|_{\mathfrak{g}_{i}}=(-1)^{i}$. The pair $(G, K)$ is a split symmetric pair. We also think of the pair $(G, K)$ concretely as $(\mathrm{SL}(V), \mathrm{SO}(V))$, where $V$ is a vector space of dimension $N$ equipped with a non-degenerate quadratic form $Q$ such that $\mathrm{SO}(V)=\mathrm{SO}(V, Q)$. We write the non-degenerate bilinear form associated to $Q$ as $\langle$,$\rangle .$

Let $\mathfrak{g}^{r s}$ denote the set of regular semisimple elements in $\mathfrak{g}$ and let $\mathfrak{g}_{1}^{r s}=\mathfrak{g}_{1} \cap \mathfrak{g}^{r s}$. Similarly, let $\mathfrak{g}^{\text {reg }}$ denote the set of regular elements in $\mathfrak{g}$ and let $\mathfrak{g}_{1}^{\text {reg }}=\mathfrak{g}_{1} \cap \mathfrak{g}^{\text {reg }}$.

Let $\mathcal{N}$ be the nilpotent cone of $\mathfrak{g}$ and let $\mathcal{N}_{1}=\mathcal{N} \cap \mathfrak{g}$. When $N$ is odd, the set of $K$-orbits in $\mathcal{N}_{1}$ is parametrized by $\mathcal{P}(N)$. When $N$ is even, the set of $\mathrm{O}(N)$-orbits in $\mathcal{N}_{1}$ is parametrized by $\mathcal{P}(N)$, moreover, each $\mathrm{O}(N)$-orbit remains one $K$-orbit if $\lambda$ has at least one odd part, and splits into two $K$-orbits otherwise. For $\lambda \in \mathcal{P}(N)$, we write $\mathcal{O}_{\lambda}$ for the corresponding nilpotent $K$-orbit in $\mathcal{N}_{1}$ when $\lambda$ has at least one odd part, and write $\mathcal{O}_{\lambda}^{\mathrm{I}}$ and $\mathcal{O}_{\lambda}^{\mathrm{II}}$ for the corresponding two nilpotent $K$-orbits in $\mathcal{N}_{1}$ when $\lambda$ has only even parts.

Let $\mathfrak{a}$ be a maximal abelian subspace of $\mathfrak{g}_{1}$. We have the "little" Weyl group

$$
W=N_{K}(\mathfrak{a}) / Z_{K}(\mathfrak{a})=S_{N} .
$$

2.3. Equivariant fundamental group and its representations. As was discussed in CVX1], the equivariant fundamental group

$$
\pi_{1}^{K}\left(\mathfrak{g}_{1}^{r s}\right) \cong Z_{K}(\mathfrak{a}) \rtimes B_{N} \cong(\mathbb{Z} / 2 \mathbb{Z})^{N-1} \rtimes B_{N},
$$

where $B_{N}$ is the braid group of $N$ strands and it acts on

$$
Z_{K}(\mathfrak{a}) \cong\left\{\left(i_{1}, \ldots, i_{N}\right) \in(\mathbb{Z} / 2 \mathbb{Z})^{N} \mid \sum_{k=1}^{N} i_{k}=0\right\} \cong(\mathbb{Z} / 2 \mathbb{Z})^{N-1}
$$

via the natural map $B_{N} \rightarrow S_{N}$. For simplicity we write

$$
\widetilde{B}_{N}=(\mathbb{Z} / 2 \mathbb{Z})^{N-1} \rtimes B_{N} \text { and } I_{N}=(\mathbb{Z} / 2 \mathbb{Z})^{N-1} .
$$

It is easy to see that the action of $B_{N}$ on $I_{N}^{\vee}$ has $[N / 2]+1$ orbits. We choose a set of representatives $\chi_{m} \in I_{N}^{\vee}, 0 \leq m \leq[N / 2]$, of the $B_{N}$-orbits as follows. Let $\tau_{i}^{\prime} \in(\mathbb{Z} / 2 \mathbb{Z})^{N}$ be 
the element with all entries 0 except the $i$-th position. Then $\left\{\tau_{i}=\tau_{i}^{\prime}+\tau_{i+1}^{\prime}, i=1, \ldots, N-1\right\}$, is a set of generators for $I_{N}$. For $0 \leq m \leq[N / 2]$, we define a character $\chi_{m}$ as follows:

$$
\chi_{m}\left(\tau_{m}\right)=-1 \text { and } \chi_{m}\left(\tau_{i}\right)=1 \text { for } i \neq m \text {. }
$$

For $\chi \in I_{N}^{\vee}$, we set

$$
B_{\chi}=\operatorname{Stab}_{B_{N}} \chi
$$

Let $s_{i}, i=1, \ldots, N-1$, be the simple reflections in $W=S_{N}$. It is easy to check that

$$
\operatorname{Stab}_{S_{N}}\left(\chi_{m}\right)=\left\langle s_{i}, i \neq m\right\rangle \cong S_{m} \times S_{N-m} \text { if } m \neq N / 2 \text {, and }
$$$$
\operatorname{Stab}_{S_{N}}\left(\chi_{m}\right) \text { contains } S_{m} \times S_{m} \text { as an index } 2 \text { normal subgroup if } m=N / 2 \text {. }
$$

Let us define

$$
B_{m, N-m}=\text { the inverse image of } S_{m} \times S_{N-m} \cong\left\langle s_{i}, i \neq m\right\rangle \text { under the map } B_{N} \rightarrow S_{N} .
$$

Then it follows from (2.2) that

$$
B_{\chi_{m}}=B_{m, N-m} \text { when } m \neq N / 2,
$$

and $B_{\chi_{m}}$ contains $B_{m, N-m}$ as an index 2 normal subgroup when $m=N / 2$.

Let $\sigma_{i}, i=1, \ldots, N-1$, be the standard generators of $B_{N}$ which are lifts of the $s_{i}$ 's under the map $B_{N} \rightarrow S_{N}$. Then $B_{m, N-m}$ is generated by $\sigma_{i}, i \neq m$, and $\sigma_{m}^{2}$. We have a natural quotient map

$$
\mathbb{C}\left[B_{m, N-m}\right] \rightarrow \mathcal{H}_{m,-1} \times \mathcal{H}_{N-m,-1} \cong \mathbb{C}\left[B_{m, N-m}\right] /\left\langle\left(\sigma_{i}-1\right)^{2}, i \neq m, \sigma_{m}^{2}-1\right\rangle .
$$

Let us write $\mathcal{H}_{m,-1} \times \mathcal{H}_{N-m,-1}=\mathcal{H}_{\chi_{m},-1}$. We consider a family of representations of $\widetilde{B}_{N}$ as follows. For $0 \leq m \leq[N / 2]$, we define

$$
L_{\chi_{m}}:=\operatorname{Ind}_{\mathbb{C}\left[B_{m, N-m}\right]}^{\mathbb{C}\left[B_{N}\right]} \mathcal{H}_{\chi_{m},-1} \cong \mathbb{C}\left[B_{N}\right] \otimes_{\mathbb{C}\left[B_{m, N-m}\right]} \mathcal{H}_{\chi_{m},-1}
$$

where in the tensor product $\mathbb{C}\left[B_{m, N-m}\right]$ acts on $\mathcal{H}_{\chi_{m},-1}$ via the quotient map (2.4) and on $\mathbb{C}\left[B_{N}\right]$ by right multiplication. The module $L_{\chi_{m}}$ has a natural $\widetilde{B}_{N}$-action defined as follows. We let $B_{N}$ act on $L_{\chi_{m}}$ by left multiplication and we let $I_{N}$ act on $L_{\chi_{m}}$ via $a .(b \otimes v)=$ $\left(\left(b \cdot \chi_{m}\right)(a)\right)(b \otimes v)$ for $a \in I_{N}, b \in B_{N}$ and $v \in \mathcal{H}_{\chi_{m},-1}$. We will view $L_{\chi_{m}}$ as a representation of the equivariant fundamental group $\widetilde{B}_{N}$ in this manner.

We will next identify the composition factors of the modules $L_{\chi_{m}}$. Let $\mu^{1} \in \mathcal{P}_{2}(m)$ and $\mu^{2} \in \mathcal{P}_{2}(N-m), m \in[0,[N / 2]]$. Proceeding just as in the definition of $L_{\chi_{m}}$, one obtains the following representation of $\widetilde{B}_{N}$ :

$$
V_{\mu^{1}, \mu^{2}}:=\operatorname{Ind}_{\mathbb{C}\left[B_{m, N-m}\right]}^{\mathbb{C}\left[B_{N}\right]}\left(D_{\mu^{1}} \otimes D_{\mu^{2}}\right) \cong \mathbb{C}\left[B_{N}\right] \otimes_{\mathbb{C}\left[B_{m, N-m}\right]}\left(D_{\mu^{1}} \otimes D_{\mu^{2}}\right) .
$$

Using (2.3) , one readily checks that $V_{\mu^{1}, \mu^{2}}$ is an irreducible representation of $\widetilde{B}_{N}$ when $m \neq$ $N / 2$, or when $m=N / 2$ and $\mu^{1} \neq \mu^{2}$. When $m=N / 2$ and $\mu^{1}=\mu^{2}, V_{\mu^{1}, \mu^{2}}$ breaks into the direct sum of two non-isomorphic irreducible representations of $\widetilde{B}_{N}$, which we denote by $V_{\mu^{1}, \mu^{2}}^{\mathrm{I}}$ and $V_{\mu^{1}, \mu^{2}}^{\mathrm{II}}$, i.e., we have

$$
V_{\mu, \mu} \cong V_{\mu, \mu}^{\mathrm{I}} \oplus V_{\mu, \mu}^{\mathrm{II}}
$$


Moreover,

$$
\begin{gathered}
\text { when } m \neq N / 2, V_{\mu^{1}, \mu^{2}} \cong V_{\nu^{1}, \nu^{2}} \text { if and only if }\left(\mu^{1}, \mu^{2}\right)=\left(\nu^{1}, \nu^{2}\right) \text {; } \\
\text { when } m=N / 2, V_{\mu^{1}, \mu^{2}} \cong V_{\nu^{1}, \nu^{2}} \text { if and only if } \\
\text { either }\left(\mu^{1}, \mu^{2}\right)=\left(\nu^{1}, \nu^{2}\right) \text { or }\left(\mu^{1}, \mu^{2}\right)=\left(\nu^{2}, \nu^{1}\right) .
\end{gathered}
$$

As the $D_{\mu^{1}} \otimes D_{\mu^{2}}$ are the composition factors of $\mathcal{H}_{\chi_{m},-1}$ we conclude:

Lemma 2.1. The composition factors of $L_{\chi_{m}}$ consist of the $V_{\mu^{1}, \mu^{2}}, \mu^{1} \neq \mu^{2}, \mu^{1} \in \mathcal{P}_{2}(m)$, $\mu^{2} \in \mathcal{P}_{2}(N-m)$, and when $N=2 m$ we have two additional composition factors $V_{\mu, \mu}^{\mathrm{I}}$ and $V_{\mu, \mu}^{\mathrm{II}}$ for $\mu \in \mathcal{P}_{2}(m)$.

\section{Maximal $\theta$-Stable PARABOliC SUbGroups AND PARABOliC INDUCTION}

Let $L$ be a $\theta$-stable Levi subgroup contained in a $\theta$-stable parabolic subgroup $P \subset G$. We write

$$
\mathfrak{l}=\text { Lie } L, \mathfrak{p}=\text { Lie } P, L_{K}=L \cap K, P_{K}=P \cap K, \mathfrak{l}_{1}=\mathfrak{l} \cap \mathfrak{g}_{1}, \mathfrak{p}_{1}=\mathfrak{p} \cap \mathfrak{g}_{1} .
$$

We will make use of the parabolic induction functor $\operatorname{Ind}_{\mathfrak{l}_{1} \subset \mathfrak{p}_{1}}^{\mathfrak{g}_{1}}: D_{L_{K}}\left(\mathfrak{l}_{1}\right) \rightarrow D_{K}\left(\mathfrak{g}_{1}\right)$ defined in $[\mathrm{H}$, L].

In this section, we study the induction functor with respect to a chosen family of $L^{m} \subset P^{m}$, $1 \leq m<N / 2$, and two more pairs $L^{n, \omega} \subset P^{n, \omega}, \omega=\mathrm{I}$, II, if $N=2 n$, where $P^{m}$ (resp. $P^{n, \omega}$ ) is a maximal $\theta$-stable parabolic subgroup and $L^{m}$ (resp. $\left.L^{n, \omega}\right)$ is a $\theta$-stable Levi subgroup of $P^{m}$ (resp. $\left.P^{n, \omega}\right)$ defined as follows.

Fix a basis $\left\{e_{i}, 1 \leq i \leq N\right\}$ of $V$ such that $\left\langle e_{i}, e_{j}\right\rangle=\delta_{i+j, N+1}$.

For $1 \leq m<N / 2$, we define $P^{m}$ to be the parabolic subgroup of $G$ that stabilizes the flag

$$
0 \subset V_{m}^{0} \subset V_{m}^{0 \perp} \subset \mathbb{C}^{N}
$$

where $V_{m}^{0}=\operatorname{span}\left\{e_{i}, 1 \leq i \leq m\right\}$. We define $L^{m}$ to be the $\theta$-stable Levi subgroup of $P^{m}$ which consists of diagonal block matrices of sizes $m, N-2 m, m$.

When $N=2 n$, for $\omega=$ I, II, we define $P^{n, \omega}$ to be the parabolic subgroup of $G$ that stabilizes the flag

$$
0 \subset V_{n}^{\omega} \subset V_{n}^{\omega \perp} \subset \mathbb{C}^{2 n}
$$

where $V_{n}^{\mathrm{I}}=\operatorname{span}\left\{e_{i}, 1 \leq i \leq n\right\}$ and $V_{n}^{\mathrm{II}}=\operatorname{span}\left\{e_{i}, 1 \leq i \leq n-1, e_{n+1}\right\}$. Let $L^{n, \omega}$ be a $\theta$-stable Levi subgroup of $P^{n, \omega}$.

According to $[\mathrm{BH}]$, every maximal $\theta$-stable parabolic subgroup of $G$ is $K$-conjugate to one of the above form.

Let $\mathfrak{p}^{m}=$ Lie $P^{m}, \mathfrak{p}_{1}^{m}=\mathfrak{p}^{m} \cap \mathfrak{g}_{1}$, and $\left(\mathfrak{n}_{P^{m}}\right)_{1}=\mathfrak{n}_{P^{m}} \cap \mathfrak{g}_{1}$, where $\mathfrak{n}_{P^{m}}$ is the nilpotent radical of $\mathfrak{p}^{m}$, etc.

Proposition 3.1. We have: 
(1) The map

$$
\pi_{m}^{N}: K \times{ }^{P_{K}^{m}}\left(\mathfrak{n}_{P^{m}}\right)_{1} \rightarrow \mathcal{N}_{1},(k, x) \mapsto \operatorname{Ad}_{k}(x)
$$

is a small map onto its image, generically one-to-one.

(2) The map

$$
\check{\pi}_{m}^{N}: K \times^{P}{ }_{K}^{m} \mathfrak{p}_{1}^{m} \rightarrow \mathfrak{g}_{1},(k, x) \mapsto \operatorname{Ad}_{k}(x) .
$$

is a small map onto its image, generically one-to-one.

The same holds for the two maps $\pi_{n}^{2 n, \omega}$ and $\check{\pi}_{n}^{2 n, \omega}$ defined using $P^{n, \omega}, \omega=\mathrm{I}$, II.

We define

$$
\mathfrak{g}_{1}^{m}=\operatorname{Im} \check{\pi}_{m}^{N}, 1 \leq m<N / 2, \mathfrak{g}_{1}^{n, \omega}=\operatorname{Im} \check{\pi}_{n}^{2 n, \omega}, \omega=\mathrm{I}, \mathrm{II} .
$$

For $m<N / 2, \mathfrak{g}_{1}^{m}$ consists of elements in $\mathfrak{g}_{1}$ with eigenvalues $a_{1}, a_{1}, \ldots, a_{m}, a_{m}, a_{j}, j \in$ $[2 m+1, N]$, where $\sum_{k=1}^{m} 2 a_{k}+\sum_{j=2 m+1}^{N} a_{j}=0$. Let

$$
\begin{gathered}
Y_{m}^{r}=\left\{x \in \mathfrak{g}_{1}^{r e g} \mid x \text { has eigenvalues } a_{1}, a_{1}, \ldots, a_{m}, a_{m}, a_{j}, j \in[2 m+1, N],\right. \\
\text { where } \left.a_{i} \neq a_{j} \text { for } i \neq j\right\} .
\end{gathered}
$$

One checks readily that $\overline{Y_{m}^{r}}=\mathfrak{g}_{1}^{m}$.

Consider the case $m=N / 2=n$. For $\omega=\mathrm{I}$, II, let

$$
\begin{gathered}
Y_{n}^{r, \omega}=\left\{x \in \mathfrak{g}_{1}^{r e g} \mid x \text { has eigenvalues } a_{1}, a_{1}, \ldots, a_{n}, a_{n}, \text { where } a_{i} \neq a_{j} \text { for } i \neq j,\right. \\
\text { and the nilpotent part of } \left.x \text { lies in the orbit } \mathcal{O}_{2^{n}}^{\omega}\right\},
\end{gathered}
$$

where $\mathcal{O}_{2^{n}}^{\omega}$ is the nilpotent orbit given by the partition $2^{m}$ and defined by the equation $\operatorname{Im} \pi_{n}^{2 n, \omega}=\overline{\mathcal{O}}_{2^{n}}^{\omega}$. Then $Y_{n}^{r, \omega}$ is an open dense subset in $\mathfrak{g}_{1}^{n, \omega}$.

Let $\left(\mathfrak{p}_{1}^{m}\right)^{r}=\mathfrak{p}_{1}^{m} \cap Y_{m}^{r}$ and $\left(\mathfrak{l}_{1}^{m}\right)^{r s}=\mathfrak{l}_{1}^{m} \cap\left(\mathfrak{l}^{m}\right)^{r s}$.

Proposition 3.2. (1) There is a natural surjective map

$$
\pi_{1}^{K}\left(Y_{m}^{r}\right) \rightarrow \pi_{1}^{L_{K}^{m}}\left(\left(\mathfrak{l}_{1}^{m}\right)^{r s}\right) \cong B_{m} \times \widetilde{B}_{N-2 m}
$$

such that for an $L_{K}^{m}$-equivariant local system $\mathcal{T}$ on $\left(\mathfrak{l}_{1}^{m}\right)^{\text {rs }}$ associated to a $\pi_{1}^{L_{K}^{m}}\left(\left(\mathfrak{l}_{1}^{m}\right)^{r s}\right)$ representation $E$, we have

$$
\operatorname{Ind}_{\mathfrak{l}_{1}^{m} \subset \mathfrak{p}_{1}^{m}}^{\mathfrak{g}_{1}} \operatorname{IC}\left(\mathfrak{l}_{1}^{m}, \mathcal{T}\right) \cong \operatorname{IC}\left(\mathfrak{g}_{1}^{m}, \mathcal{T}^{\prime}\right)
$$

where $\mathcal{T}^{\prime}$ is the $K$-equivariant local system on $Y_{m}^{r}$ associated to the representation of $\pi_{1}^{K}\left(Y_{m}^{r}\right)$ which is obtained from E by pull-back under the map (3.2).

(2) We have a natural surjective map

$$
\pi_{1}^{K}\left(Y_{n}^{r, \omega}\right) \rightarrow \pi_{1}^{L_{K}^{n, \omega}}\left(\left(\mathfrak{l}_{1}^{n, \omega}\right)^{r s}\right) \cong B_{n}, \omega=\mathrm{I}, \mathrm{II},
$$

such that for an $L_{K}^{n, \omega}$-equivariant local system $\mathcal{T}$ on $\left(\mathfrak{l}_{1}^{n, \omega}\right)^{r s}$ associated to a $\pi_{1}^{L_{K}^{n, \omega}}\left(\left(L_{1}^{n, \omega}\right)^{r s}\right)$ representation $E$, we have

$$
\operatorname{Ind}_{\mathfrak{l}_{1}^{n, \omega} \subset \mathfrak{p}_{1}^{n, \omega}}^{\mathfrak{g}_{1}} \operatorname{IC}\left(\mathfrak{l}_{1}^{n, \omega}, \mathcal{T}\right) \cong \operatorname{IC}\left(\mathfrak{g}_{1}^{n, \omega}, \mathcal{T}^{\prime}\right)
$$


where $\mathcal{T}^{\prime}$ is the $K$-equivariant local system on $Y_{n}^{r, \omega}$ associated to the representation of $\pi_{1}^{K}\left(Y_{n}^{r, \omega}\right)$ which is obtained from $E$ by pull-back under the map (3.3).

3.1. Proof of Proposition 3.1. We begin with the proof of (1). Consider the following projection

$$
\tau_{m}^{N}:\left\{\left(x, 0 \subset V_{m} \subset V_{m}^{\perp} \subset V=\mathbb{C}^{N}\right) \mid x \in \mathfrak{g}_{1}, x V_{m}=0, x V_{m}^{\perp} \subset V_{m}\right\} \rightarrow \mathcal{N}_{1} .
$$

When $m \neq N / 2$, the map $\tau_{m}^{N}$ can be identified with the map $\pi_{m}^{N}$. When $N=2 m$, the image of the map $\tau_{m}^{2 m}$ has two irreducible components, i.e., closures of the two orbits $\mathcal{O}_{2^{m}}^{\mathrm{I}}$ and $\mathcal{O}_{2^{m}}^{\mathrm{II}}$. The two maps $\pi_{m}^{N \text {,I }}$ and $\pi_{m}^{N \text {,II }}$ can be identified with the map $\tau_{m}^{2 m}$ restricted to the inverse image of $\overline{\mathcal{O}}_{2^{m}}^{\mathrm{I}}$ and $\overline{\mathcal{O}}_{2^{m}}^{\mathrm{II}}$ respectively. Thus it suffices to show that

$$
\text { the map } \tau_{m}^{N} \text { is small over its image and generically one-to-one. }
$$

When $m \neq N / 2$, one can check that the image of $\tau_{m}^{N}$ is as follows

$$
\operatorname{Im} \tau_{m}^{N}=\overline{\mathcal{O}}_{3^{m} 1^{N-3 m}} \text { if } N \geq 3 m, \quad \operatorname{Im} \tau_{m}^{N}=\overline{\mathcal{O}}_{3^{N-2 m} 2^{3 m-N}} \text { if } N<3 m .
$$

Assume that $N \geq 3 m$ and $x \in \mathcal{O}_{3^{m} 1^{N-3 m}}$. Then $\left(\tau_{m}^{N}\right)^{-1}(x)=\operatorname{Im} x^{2}$. Assume that $N<3 m$ and $x \in \mathcal{O}_{3^{N-2 m} 2^{3 m-N}}$. Then $\left(\tau_{m}^{N}\right)^{-1}(x)=\operatorname{ker} x$. This proves that $\tau_{m}^{N}$ is generically one-toone.

Let $x \in \mathcal{O}_{3^{i} 2^{j} 1^{N-3 i-2 j}} \subset \operatorname{Im} \tau_{m}^{N}$. We assume that $3^{i} 2^{j} 1^{N-3 i-2 j} \neq 3^{m} 1^{N-3 m}$ if $N \geq 3 m$, and $3^{i} 2^{j} 1^{N-3 i-2 j} \neq 3^{N-2 m} 2^{3 m-N}$ if $N<3 m$. It suffices to show that

$$
\operatorname{dim}\left(\tau_{m}^{N}\right)^{-1}(x)<\operatorname{codim}_{\operatorname{Im} \tau_{m}^{N}} \mathcal{O}_{3^{i} 2^{j} 1^{N-3 i-2 j}} / 2 .
$$

Let $x_{0} \in \mathcal{O}_{2^{j} 1^{N-3 i-2 j}} \subset \operatorname{Im} \tau_{m-i}^{N-3 i}$. (Note that $\tau_{m-i}^{N-3 i}$ is defined since $m-i \leq(N-3 i) / 2$.) One checks readily that

$$
\left(\tau_{m}^{N}\right)^{-1}(x) \cong\left(\tau_{m-i}^{N-3 i}\right)^{-1}\left(x_{0}\right) \text { and } \operatorname{codim}_{\operatorname{Im} \tau_{m}^{N}} \mathcal{O}_{3^{i} 2^{j} 1^{N-3 i-2 j}}=\operatorname{codim}_{\operatorname{Im} \tau_{m-i}^{N-3 i}} \mathcal{O}_{2^{j} 1^{N-3 i-2 j}} .
$$

Thus it suffices to show that

$$
\operatorname{dim}\left(\tau_{m-i}^{N-3 i}\right)^{-1}\left(x_{0}\right)<\operatorname{codim}_{\operatorname{Im} \tau_{m-i}^{N-3 i}} \mathcal{O}_{2^{j} 1^{N-3 i-2 j}} / 2
$$

Let us write

$$
\begin{gathered}
\Omega_{m, j}^{N}=\left(\tau_{m}^{N}\right)^{-1}\left(\zeta_{j}\right) \text { for } \zeta_{j} \in \mathcal{O}_{2^{j} 1^{N-2 j}} \subset \operatorname{Im} \tau_{m}^{N} \\
\text { and } a_{m, j}^{N}=\operatorname{codim}_{\operatorname{Im} \pi_{m}^{N}} \mathcal{O}_{2^{j} 1^{N-2 j}}=m(2 N-3 m)-j(N-j) .
\end{gathered}
$$

To prove that the map $\tau_{m}^{N}$ is small, we are reduced to proving that

$$
\operatorname{dim} \Omega_{m, j}^{N}<\frac{a_{m, j}^{N}}{2}
$$

To prove this we recall the partitioning of $\Omega_{m, j}^{N}$ into $\Omega_{m, j}^{N, k}$ given in [CVX2, Section2] as follows:

$$
\Omega_{m, j}^{N, k}=\left\{\left(0 \subset V_{m} \subset V_{m}^{\perp} \subset V=\mathbb{C}^{N}\right) \mid \operatorname{dim}\left(V_{m} \cap \zeta_{j} V\right)=k\right\} .
$$

We have

$$
\Omega_{m, j}^{N, k} \neq \emptyset \Leftrightarrow \max \{m+j-N / 2, j / 2\} \leq k \leq \min \{j, m\}
$$


Recall that we have a surjective map $\Omega_{m, j}^{N, k} \rightarrow \operatorname{OGr}(j-k, j) \times \operatorname{OGr}(m-k, N-2 j)$ with fibers being affine spaces $\mathbb{A}^{(m-k)(j-k)}$. We have

$$
\operatorname{dim} \overline{\Omega_{m, j}^{N, k}}=-2 k^{2}+(-N+3 j+2 m+1) k+m N-m j-\frac{j^{2}+3 m^{2}+j+m}{2} .
$$

One checks that

$$
\begin{gathered}
\text { if } j \geq N-2 m, \operatorname{dim} \overline{\Omega_{m, j}^{N, k}} \text { is maximal when } k=m+j-\left[\frac{N}{2}\right], \\
\text { if } j<N-2 m, \operatorname{dim} \overline{\Omega_{m, j}^{N, k}} \text { is maximal when } k=\left[\frac{j+1}{2}\right] .
\end{gathered}
$$

Thus a direct calculation shows that

$\operatorname{dim}\left(\pi_{m}^{N}\right)^{-1}\left(\zeta_{j}\right)=\left\{\begin{array}{cl}\frac{a_{m, j}^{N}}{2}+\frac{j+m-N}{2} & \text { if } j \geq N-2 m \text { and } N \text { even, or } j<N-2 m \text { and } j \text { odd } \\ \frac{a_{m, j}^{N}}{2}-\frac{m}{2} & \text { if } j \geq N-2 m \text { and } N \text { odd, or } j<N-2 m \text { and } j \text { even. }\end{array}\right.$

This proves (3.5) (note that $m+j<N)$. The proof of (3.4) is complete. This finishes the proof of the claim (1) in the proposition.

It then follows that we have

$$
\left(\pi_{m}^{N}\right)_{*} \mathbb{C}[-] \cong \operatorname{IC}\left(\overline{\mathcal{O}}_{\lambda}, \mathbb{C}\right),\left(\operatorname{resp} .\left(\left(\pi_{N / 2}^{N}\right)^{\omega}\right)_{*} \mathbb{C}[-] \cong \operatorname{IC}\left(\overline{\mathcal{O}}_{\lambda}^{\omega}, \mathbb{C}\right), \omega=\mathrm{I}, \mathrm{II},\right)
$$

where

$$
\lambda=3^{m} 1^{N-3 m} \text { if } N \geq 3 m, \quad \lambda=3^{N-2 m} 2^{3 m-N} \text { if } N<3 m .
$$

Note that $K \times{ }^{P_{K}^{m}} \mathfrak{p}_{1}^{m}$ is the orthogonal complement of $K \times{ }_{K}^{m}\left(\mathfrak{n}_{P^{m}}\right)_{1}$ in the trivial bundle $K \times \mathfrak{g}_{1}$ over $K / P_{K}^{m}$. By the functoriality of Fourier transform, we have that

$$
\mathfrak{F}\left(\left(\pi_{m}^{N}\right)_{*} \mathbb{C}[-]\right) \cong\left(\check{\pi}_{m}^{N}\right)_{*} \mathbb{C}[-] .
$$

Since Fourier transform sends simple perverse sheaves to simple perverse sheaves, we can conclude from (3.6) and (3.7) that

$$
\left(\check{\pi}_{m}^{N}\right)_{*} \mathbb{C}[-] \cong \mathrm{IC}\left(\operatorname{Im} \check{\pi}_{m}^{N}, \mathbb{C}\right) .
$$

This proves the claim (2) of the proposition. The argument for $\left(\check{\pi}_{n}^{2 n}\right)^{\omega}, \omega=\mathrm{I}$, II, is the same. The proof of the proposition is complete.

3.2. Proof of Proposition 3.2. Recall that the parabolic induction functor

$$
\operatorname{Ind}_{\mathfrak{l}_{1} \subset \mathfrak{p}_{1}}^{\mathfrak{g}_{1}}: D_{L_{K}}\left(\mathfrak{l}_{1}\right) \rightarrow D_{K}\left(\mathfrak{g}_{1}\right)
$$

can be defined as follows $([\underline{\mathrm{H}}, \underline{\mathrm{L}}])$. Let

$$
\operatorname{pr}: \mathfrak{p}_{1}=\mathfrak{l}_{1} \oplus\left(\mathfrak{n}_{P}\right)_{1} \rightarrow \mathfrak{l}_{1}
$$

be the natural projection map, where $\mathfrak{n}_{P}$ is the nilpotent radical of $\mathfrak{p}$. Consider the diagram

$$
\mathfrak{l}_{1} \stackrel{\mathrm{pr}}{\longleftarrow} \mathfrak{p}_{1} \stackrel{p_{1}}{\longleftarrow} K \times \mathfrak{p}_{1} \stackrel{p_{2}}{\longrightarrow} K \times^{P_{K}} \mathfrak{p}_{1} \stackrel{\check{\pi}}{\longrightarrow} \mathfrak{g}_{1} .
$$

The maps in (3.8) are $K \times P_{K}$-equivariant, where $K$ acts trivially on $\mathfrak{l}_{1}, \mathfrak{p}_{1}$, by left multiplication on the $K$-factor on $K \times \mathfrak{p}_{1}$ and on $K \times^{P_{K}} \mathfrak{p}_{1}$, and by adjoint action on $\mathfrak{g}_{1}$, and $P_{K}$ acts on $\mathfrak{l}_{1}$ by $a . l=\operatorname{pr}(\operatorname{Ad} a(l))$, by adjoint action on $\mathfrak{p}_{1}$, by $a .(k, p)=\left(k a^{-1}, \operatorname{Ad} a(p)\right)$ on $K \times \mathfrak{p}_{1}$, 
trivially on $K \times{ }^{P_{K}} \mathfrak{p}_{1}$ and on $\mathfrak{g}_{1}$. Let $A$ be a complex in $D_{L_{K}}\left(\mathfrak{l}_{1}\right)$. Then $\left(\operatorname{pr} \circ p_{1}\right)^{*} A \cong p_{2}^{*} A^{\prime}$ for a well-defined complex $A^{\prime}$ in $D_{K}\left(K \times{ }^{P_{K}} \mathfrak{p}_{1}\right)$. Define

$$
\operatorname{Ind}_{\mathfrak{l}_{1} \subset \mathfrak{p}_{1}}^{\mathfrak{g}_{1}} A=\check{\pi}_{!} A^{\prime}[\operatorname{dim} P-\operatorname{dim} L] .
$$

It is shown in $[\mathrm{H}, \mathrm{L}]$ that the induction functor commutes with Fourier transform, i.e.,

$$
\mathfrak{F}\left(\operatorname{Ind}_{\mathfrak{l}_{1} \subset \mathfrak{p}_{1}}^{\mathfrak{g}_{1}} A\right) \cong \operatorname{Ind}_{\mathfrak{l}_{1} \subset \mathfrak{p}_{1}}^{\mathfrak{g}_{1}}(\mathfrak{F}(A)) .
$$

Note that we have that

$$
L_{K}^{m} \cong \mathrm{GL}(m) \times \mathrm{SO}(N-2 m) \text { and }\left(\mathfrak{l}^{m}\right)_{1} \cong \mathfrak{g l}(m) \oplus \mathfrak{s l}(N-2 m)_{1} .
$$

To ease notations, let us write now that $L=L^{m}, P=P^{m}$, and $\check{\pi}=\check{\pi}_{m}^{N}$ etc.

We first show that

The map $\check{\pi}\left(\operatorname{resp} . \check{\pi}_{n}^{\omega}\right)$, when restricted to $\check{\pi}^{-1}\left(Y^{r}\right)\left(\operatorname{resp} . \check{\pi}^{-1}\left(Y_{n}^{r, \omega}\right)\right)$,

is one-to-one.

Each element in $Y^{r}$ is $K$-conjugate to an element $x_{0} \in \mathfrak{p}_{1}$ (see [KR, Theorem 7$]$ ), where

$$
\begin{gathered}
x_{0} e_{i}=a_{i} e_{i}, x_{0} e_{N+1-i}=e_{i}+a_{i} e_{N+1-i} \text { for } i \in[1, m], \\
x_{0} e_{j}=b_{j} e_{j}+c_{j} e_{N+1-j}, x_{0} e_{2 n+2-j}=c_{j} e_{j}+b_{j} e_{N+1-j} \text { for } j \in[m+1,[N / 2]] \\
x_{0} e_{(N+1) / 2}=b_{(N+1) / 2} e_{(N+1) / 2} \text { if } N \text { is odd }
\end{gathered}
$$

and the numbers $a_{i}, i=1, \ldots, m, b_{j}+c_{j}, b_{j}-c_{j}, j=m+1, \ldots,[N / 2], b_{(N+1) / 2}$ are distinct.

It suffices to show that $\check{\pi}^{-1}\left(x_{0}\right)$ consists of one point. Note that $\check{\pi}^{-1}\left(x_{0}\right)$ consists of $x_{0^{-}}$ stable $m$-dimensional isotropic subspaces of $V$. It is clear that $U^{0}:=\operatorname{span}\left\{e_{1}, \ldots, e_{m}\right\} \in$ $\check{\pi}^{-1}\left(x_{0}\right)$. Assume that $U_{m} \in \check{\pi}^{-1}\left(x_{0}\right)$. We can extend $U_{m}$ to a complete flag that is $x_{0}$-stable. Since $x_{0}$ is regular, all $x_{0}$-stable flags are in one $W$-orbit. Thus there exists $w \in W$ such that $U_{m}=w U^{0}$. If $U_{m} \neq U^{0}$, then there exists a $e_{i}, i \in[1, m]$, such that $w e_{i}=e_{j}$ and $j \notin[1, m]$. Then we have either $\left\langle x_{0} e_{j}, e_{j}\right\rangle \neq 0$ or $\left\langle e_{j}, e_{j}\right\rangle \neq 0$. But both $x_{0} e_{j}$ and $e_{j}$ are in $U_{m}$. This contradicts the fact that $U_{m}$ is isotropic. This proves (3.11) for $\check{\pi}_{m}, m<N / 2$. The proof for $\check{\pi}_{n}^{\omega}$ is entirely similar and omitted.

Now we show that

The image of $\mathfrak{p}_{1}^{r}$ under the map pr: $\mathfrak{p}_{1} \rightarrow \mathfrak{l}_{1}$ is $\mathfrak{l}_{1}^{r s}$.

Let $x \in \mathfrak{p}_{1}^{r}$. By the above proof of (3.11) we can assume that $\operatorname{Ad}(k) x=x_{0}$ for some $k \in K$, where $x_{0}$ is as in (3.12). Thus $(k, x) \in \check{\pi}^{-1}\left(x_{0}\right)$. It follows from (3.11) that $(k, x)=\left(1, x_{0}\right) \in$ $K \times{ }^{P_{K}} \mathfrak{p}_{1}$. Hence $k \in P_{K}$. Assume that $k=l u$ where $l \in L_{K}$ and $u \in U_{K}=U \cap K(U$ is the unipotent radical of $P)$. Then we have $\operatorname{pr}(x)=\operatorname{pr}\left(\operatorname{Ad}\left(u^{-1} l^{-1}\right) x_{0}\right)=\operatorname{pr}\left(\operatorname{Ad}\left(l^{-1}\right) x_{0}\right)=$ $\operatorname{Ad}\left(l^{-1}\right) \operatorname{pr}\left(x_{0}\right)$. It is clear that $\operatorname{pr}\left(x_{0}\right) \in \mathfrak{l}^{r s}$. Thus $(3.13)$ follows.

By (3.11) and (3.13), we have the following diagram, when restricting (3.8) to $Y^{r}$,

$$
\mathfrak{l}_{1}^{r s} \stackrel{\mathrm{pr}}{\longleftarrow} \mathfrak{p}_{1}^{r} \stackrel{p_{1}}{\longleftarrow} K \times \mathfrak{p}_{1}^{r} \stackrel{p_{2}}{\longrightarrow} K \times^{P_{K}} \mathfrak{p}_{1}^{r} \stackrel{\check{\pi}}{\longrightarrow} Y^{r}
$$


Using (3.11), we see that

$$
\pi_{1}^{K}\left(Y^{r}\right) \cong \pi_{1}^{K \times P_{K}}\left(Y^{r}\right) \cong \pi_{1}^{K \times P_{K}}\left(K \times{ }^{P_{K}} \mathfrak{p}_{1}^{r}\right) \cong \pi_{1}^{K \times P_{K}}\left(K \times \mathfrak{p}_{1}^{r}\right) \cong \pi_{1}^{P_{K}}\left(\mathfrak{p}_{1}^{r}\right) .
$$

Finally, the canonical map $\pi_{1}^{P_{K}}\left(\mathfrak{p}_{1}^{r}\right) \rightarrow \pi_{1}^{P_{K}}\left(\mathfrak{l}_{1}^{r s}\right) \cong \pi_{1}^{L_{K}}\left(\mathfrak{l}_{1}^{r s}\right)$ is surjective. We see this as follows. First, the canonical map above can be identified with the canonical map $\pi_{1}^{P_{K}}\left(\mathfrak{p}_{1}^{r}\right) \rightarrow$ $\pi_{1}^{P_{K}}\left(\mathrm{pr}^{-1}\left(\mathfrak{l}_{1}^{r s}\right)\right)$. Now, because $\mathfrak{p}_{1}^{r}$ is an open subset in $\operatorname{pr}^{-1}\left(\mathfrak{l}_{1}^{r s}\right)$, which is smooth, the map $\pi_{1}\left(\mathfrak{p}_{1}^{r}\right) \rightarrow \pi_{1}\left(\mathrm{pr}^{-1}\left(\mathfrak{l}_{1}^{r s}\right)\right)$ is a surjection. To conclude that this property persists when we pass to the equivariant fundamental group it suffices to remark that the equivariant fundamental group is always a quotient of the ordinary fundamental group as long as the group is connected. We now conclude the argument making use of Proposition 3.1.

\section{Fourier transform of nilpotent orbital Complexes for $(\operatorname{SL}(N), \mathrm{SO}(N))$}

Consider the symmetric pair $(G, K)=(\mathrm{SL}(N), \mathrm{SO}(N))$. Let us write $\mathcal{A}_{N}$ for the set of all simple $K$-equivariant perverse sheaves on $\mathcal{N}_{1}$ (up to isomorphism), that is, the set of IC complexes $\operatorname{IC}(\mathcal{O}, \mathcal{E})$, where $\mathcal{O}$ is a $K$-orbit in $\mathcal{N}_{1}$ and $\mathcal{E}$ is an irreducible $K$-equivariant local system on $\mathcal{O}$ (up to isomorphism). An IC complex in $\mathcal{A}_{N}$ is called a nilpotent orbital complex.

Let $n=[N / 2]$. We set

$$
\begin{gathered}
\Sigma_{N}=\left\{\left(\nu ; \mu^{1}, \mu^{2}\right) \mid 0 \leq m \leq n, \nu \in \mathcal{P}(m)\right. \\
\left.0 \leq k \leq n-m, \mu^{1} \in \mathcal{P}_{2}(k), \mu^{2} \in \mathcal{P}_{2}(N-2 m-k)\right\} .
\end{gathered}
$$

In the case when $N$ is even, we identify the triple $\left(\nu ; \mu^{1}, \mu^{2}\right)$ with $\left(\nu ; \mu^{2}, \mu^{1}\right)$ if $\left|\mu^{1}\right|=\left|\mu^{2}\right|$ and $\mu^{1} \neq \mu^{2}$, and the triples $(\nu ; \mu, \mu)$ attain two labels I and II.

Given a triple $\left(\nu ; \mu^{1}, \mu^{2}\right) \in \Sigma_{N}$ (resp. $\left.(\nu ; \mu, \mu)^{\omega} \in \Sigma_{N}, \omega=\mathrm{I}, \mathrm{II}\right)$, where $|\nu|=m<N / 2$, we define an irreducible $K$-equivariant local system $\mathcal{T}\left(\nu ; \mu^{1}, \mu^{2}\right)\left(\operatorname{resp} . \mathcal{T}(\nu ; \mu, \mu)^{\omega}\right)$ on $Y_{m}^{r}$ (here we write $Y_{0}^{r}=\mathfrak{g}_{1}^{r s}$ ) as follows. We obtain a map

$$
\tau: \pi_{1}^{K}\left(Y_{m}^{r}\right) \rightarrow B_{m} \times \widetilde{B}_{N-2 m} \rightarrow S_{m} \times \widetilde{B}_{N-2 m}
$$

by composing the map in (3.2) with the natural map $B_{m} \times \widetilde{B}_{N-2 m} \rightarrow S_{m} \times \widetilde{B}_{N-2 m}$. Note that the map $\tau$ is surjective. Then $\mathcal{T}\left(\nu ; \mu^{1}, \mu^{2}\right)$ (resp. $\left.\mathcal{T}(\nu ; \mu, \mu)^{\omega}\right)$ is the irreducible local syste associated to the irreducible representation of $\pi_{1}^{K}\left(Y_{m}^{r}\right)$ given by pulling back the irreducible representation $\rho_{\nu} \otimes V_{\mu^{1}, \mu^{2}}$ (resp. $\rho_{\nu} \otimes V_{\mu, \mu}^{\omega}$ ) via the map $\tau$; here $\rho_{\nu} \in S_{m}^{\vee}$ is the irreducible representation of $S_{m}$ corresponding to $\nu \in \mathcal{P}(m)$ and $V_{\mu^{1}, \mu^{2}}$ (resp. $V_{\mu, \mu}^{\omega}$ ) is the irreducible representation of $\widetilde{B}_{N-2 m}$ defined in (2.5) (resp. (2.7)).

Assume now that $N=2 n$. Given a triple $(\nu ; \emptyset, \emptyset)^{\omega} \in \Sigma_{N}, \omega=$ I, II, we define the irreducible $K$-equivariant local system $\mathcal{T}(\nu ; \emptyset, \emptyset)^{\omega}$ on $\left(Y_{n}^{r}\right)^{\omega}$ as the local system associated to the representation of $\pi_{1}^{K}\left(\left(Y_{n}^{r}\right)^{\omega}\right)$ obtained by pulling back the representation $\rho_{\nu} \in S_{n}^{\vee}$ corresponding to $\nu \in \mathcal{P}(n)$ under that map

$$
\pi_{1}^{K}\left(\left(Y_{n}^{r}\right)^{\omega}\right) \rightarrow B_{n} \rightarrow S_{n} .
$$


Now we are ready to formulate our main result:

Theorem 4.1. The Fourier transform $\mathfrak{F}: \operatorname{Perv}_{K}\left(\mathfrak{g}_{1}\right) \rightarrow \operatorname{Perv}_{K}\left(\mathfrak{g}_{1}\right)$ induces a bijection

$$
\begin{aligned}
\mathfrak{F} & : \mathcal{A}_{N} \stackrel{\sim}{\rightarrow}\left\{\operatorname{IC}\left(\mathfrak{g}_{1}^{m}, \mathcal{T}\left(\nu ; \mu^{1}, \mu^{2}\right)\right)\left|\left(\nu ; \mu^{1}, \mu^{2}\right) \in \Sigma_{N}, \mu^{1} \neq \mu^{2},\right| \nu \mid=m<N / 2\right\} \\
& \cup\left\{\operatorname{IC}\left(\mathfrak{g}_{1}^{m}, \mathcal{T}(\nu ; \mu, \mu)^{\omega}\right)\left|(\nu ; \mu, \mu)^{\omega} \in \Sigma_{N}, \omega=\mathrm{I}, \mathrm{II},\right| \nu \mid=m<N / 2\right\} \text { (if } N \text { is even), } \\
& \cup\left\{\operatorname{IC}\left(\mathfrak{g}_{1}^{n, \omega}, \mathcal{T}(\nu ; \emptyset, \emptyset)^{\omega}\right)\left|(\nu ; \emptyset, \emptyset)^{\omega} \in \Sigma_{N}, \omega=\mathrm{I}, \mathrm{II},\right| \nu \mid=n=N / 2\right\} \text { (if } N \text { is even), }
\end{aligned}
$$

where $\mathfrak{g}_{1}^{0}=\mathfrak{g}_{1}, \mathfrak{g}_{1}^{m}$ and $\mathfrak{g}_{1}^{n, \omega}$ are defined in (3.1).

4.1. Proof of Theorem 4.1. Let $p(k)$ denote the number of partitions of $k$ and let $q(k)$ denote the number of 2-regular partitions of $k$. We write $p(0)=q(0)=1$. Let us define

$$
\begin{gathered}
d(k)=\sum_{s=0}^{k} q(s) q(2 k+1-s), \\
e(k)=\sum_{s=0}^{k-1} q(s) q(2 k-s)+\frac{q(k)^{2}+3 q(k)}{2} .
\end{gathered}
$$

Lemma 4.2. We have

$$
\begin{aligned}
\left|\mathcal{A}_{2 n+1}\right| & =\sum_{k=0}^{n} p(n-k) d(k)=\left|\Sigma_{2 n+1}\right| \\
\left|\mathcal{A}_{2 n}\right| & =\sum_{k=0}^{n} p(n-k) e(k)=\left|\Sigma_{2 n}\right| .
\end{aligned}
$$

Proof. Note that

$$
\sum_{k \geq 0} p(k) x^{k}=\prod_{s \geq 1} \frac{1}{1-x^{s}} \text { and } \sum_{k \geq 0} q(k) x^{k}=\prod_{s \geq 1}\left(1+x^{s}\right) .
$$

Let $p(l, k)$ denote the number of partitions of $l$ into (not necessarily distinct) parts of exactly $k$ different sizes. We have (see for example [GS])

$$
\sum_{l, k \geq 0} p(l, k) x^{l} y^{k}=\prod_{s \geq 1}\left(1+\frac{y x^{s}}{1-x^{s}}\right) .
$$

Assume first that $N=2 n+1$. Note that if $\lambda$ is a partition of $N$ with parts of $k$ different sizes, then the component group $A_{K}(x)$ of the centralizer $Z_{K}(x)$ for $x \in \mathcal{O}_{\lambda}$ is $(\mathbb{Z} / 2 \mathbb{Z})^{k-1}$. Thus there are $2^{k-1}$ irreducible $K$-equivariant local systems on $\mathcal{O}_{\lambda}$ (up to isomorphism). Hence using (4.6), we see that

$$
\left|\mathcal{A}_{2 n+1}\right|=\sum_{k \geq 0} p(2 n+1, k) 2^{k-1}=\text { Coefficient of } x^{2 n+1} \text { in } \frac{1}{2} \prod_{s \geq 1}\left(\frac{1+x^{s}}{1-x^{s}}\right) .
$$


Using (4.5), we see that

$$
\prod_{s \geq 1}\left(\frac{1+x^{s}}{1-x^{s}}\right)=\left(\sum_{k \geq 0} p(k) x^{2 k}\right)\left(\sum_{k \geq 0} q(k) x^{k}\right)^{2} .
$$

It then follows that $\left|\mathcal{A}_{2 n+1}\right|$ is the desired number. The fact that $\left|\Sigma_{2 n+1}\right|$ equals the same number is clear from the definition. Thus (4.3) holds.

Assume now that $N=2 n$. Suppose that $\lambda$ is a partition of $N$ with parts of exactly $k$ different sizes. If $\lambda$ has at least one odd part, then there are $2^{k-1}$ irreducible $K$-equivariant local systems on $\mathcal{O}_{\lambda}$ (up to isomorphism). If $\lambda$ has only even parts, then there are $2^{k}$ irreducible $K$-equivariant local systems on each $\mathcal{O}_{\lambda}^{\omega}$ (up to isomorphism), $\omega=$ I, II.

Thus we have that

$$
\begin{gathered}
\left|\mathcal{A}_{2 n}\right|=\sum_{k \geq 0} p(2 n, k) 2^{k-1}+\sum_{k \geq 0} p(n, k) 3 \cdot 2^{k-1} \\
=\text { Coefficient of } x^{2 n} \text { in } \frac{1}{2} \prod_{s \geq 1}\left(\frac{1+x^{s}}{1-x^{s}}\right)+\text { Coefficient of } x^{n} \text { in } \frac{3}{2} \prod_{s \geq 1}\left(\frac{1+x^{s}}{1-x^{s}}\right) \\
=\frac{1}{2}\left(\sum_{k=0}^{n} p(n-k)\left(2 \sum_{s=0}^{k-1} q(s) q(2 k-s)+q(k)^{2}\right)\right)+\frac{3}{2} \sum_{k=0}^{n} p(n-k) q(k)=\sum_{k=0}^{n} p(n-k) e(k) .
\end{gathered}
$$

Here we have used (4.7) and the following equation

$$
\prod_{s \geq 1}\left(\frac{1+x^{s}}{1-x^{s}}\right)=\left(\sum_{k \geq 0} p(k) x^{k}\right)\left(\sum_{k \geq 0} q(k) x^{k}\right) .
$$

Again the fact that $\left|\Sigma_{2 n}\right|$ equals the desired number is clear from the definition.

Note that the IC sheaves appearing on the right hand side of the Fourier transform map $\mathfrak{F}$ in Theorem 4.1 are pairwise non-isomorphic. Thus, in view of Lemma 4.2, Theorem 4.1 follows from:

Proposition 4.3. Let $\left(\nu ; \mu^{1}, \mu^{2}\right) \in \Sigma_{N}$ (resp. $(\nu ; \mu, \mu)^{\omega} \in \Sigma_{N}, \omega=\mathrm{I}$, II) and write $m=|\nu|$. The Fourier transform of $\operatorname{IC}\left(\mathfrak{g}_{1}^{m}, \mathfrak{T}\left(\nu ; \mu^{1}, \mu^{2}\right)\right)$ (resp. $\left.\operatorname{IC}\left(\mathfrak{g}_{1}^{m}, \mathcal{T}(\nu ; \mu, \mu)^{\omega}\right), \operatorname{IC}\left(\mathfrak{g}_{1}^{n, \omega}, \mathcal{T}(\nu ; \emptyset, \emptyset)^{\omega}\right)\right)$ is supported on a $K$-orbit in $\mathcal{N}_{1}$.

Proof. Let $n=[N / 2]$. We begin the proof by showing that for $\left(\emptyset ; \mu^{1}, \mu^{2}\right) \in \Sigma_{N}$ (resp. $\left.(\emptyset ; \mu, \mu)^{\omega} \in \Sigma_{N}, \omega=\mathrm{I}, \mathrm{II}\right)$

The Fourier transform of IC $\left(\mathfrak{g}_{1}, \mathcal{T}\left(\emptyset ; \mu^{1}, \mu^{2}\right)\right)$ (resp. IC $\left.\left(\mathfrak{g}_{1}, \mathfrak{T}(\emptyset ; \mu, \mu)^{\omega}\right)\right)$ is supported on a $K$-orbit in $\mathcal{N}_{1}$.

Recall that $\left.\mathfrak{T}\left(\emptyset ; \mu^{1}, \mu^{2}\right)\right)$ (resp. $\left.\mathcal{T}(\emptyset ; \mu, \mu)^{\omega}\right)$ is the irreducible $K$-equivariant local system on $\mathfrak{g}_{1}^{r s}$ corresponding to $V_{\mu^{1}, \mu^{2}}\left(\right.$ resp. $\left.V_{\mu, \mu}^{\omega}\right)$. 
We make use of a slight extension of Grinberg's work [G1, G2]. This extension is due to Grinberg who communicated it to us. Consider the adjoint quotient map $f: \mathfrak{g}_{1} \rightarrow \mathfrak{g}_{1} / / K \cong$ $\mathfrak{a} / W$. The elements $\chi \in I_{N}^{\vee}$ correspond to $K$-equivariant local systems $\mathcal{L}_{\chi}$ on $\mathfrak{g}_{1}^{r s}$. Let us write $P_{\chi} \in \operatorname{Perv}_{K}\left(\mathcal{N}_{1}\right)$ for the nearby cycle sheaf for the family $f: \mathfrak{g}_{1} \rightarrow \mathfrak{g}_{1} / / K \cong \mathfrak{a} / W$ with coefficients in the local system $\mathcal{L}_{\chi}$. Note that in order to perform this construction we have to pass to a cover of $\mathfrak{a} / W$ if $\chi$ is non-trivial. Also, observe that $P_{\chi} \cong P_{b . \chi}$ for $b \in B_{N}$.

Let us consider the $\chi_{m}, 0 \leq m \leq n$, defined in (2.1). We write $\mathcal{L}_{\chi_{m}}$ for the $K$-equivariant local system on $\mathfrak{g}_{1}^{r s}$ corresponding to the representation $L_{\chi_{m}}$ of $\pi_{1}^{K}\left(\mathfrak{g}_{1}^{r s}\right)=\widetilde{B}_{N}$ defined in (2.6) .

Theorem 4.4. (Grinberg) We have

$$
\mathfrak{F}\left(\mathcal{P}_{\chi_{m}}\right) \cong \operatorname{IC}\left(\mathfrak{g}_{1}, \mathcal{L}_{\chi_{m}}\right)
$$

In [G2, Theorem 6.1] this statements is proved when $m=0$, i.e., in the case of the trivial local system $\mathcal{L}_{\chi_{0}}$. To treat the general case only a few changes to the argument are necessary. We explain these modification briefly. Note that in G2] Grinberg works in the more general context of polar representations. In this context [G2, Theorem 6.1] is a direct application of [G2, Theorem 5.2] and an explicit, well-known, calculation in the case of quadrics in $\mathbb{C}^{k}$, i.e. the case of $S O(k)$ acting on $\mathbb{C}^{k}$. In our case $k=2$. To extend this argument to local systems $\mathcal{L}_{\chi_{m}}$ we have to insert the local system into the Picard-Lefschetz construction of an explicit basis of $\mathfrak{F}\left(\mathcal{P}_{\chi_{m}}\right)_{\ell}$ at a chosen base point $\ell \in \mathfrak{g}_{1}^{r s}$ as in section 4 of [G2]. Once this is done we can again reduce the result to an explicit calculation in the case of $S O(2)$ acting on $\mathbb{C}^{2}$ but this time with a -1-local system on the the regular semisimple points.

By Lemma 2.1 the IC sheaves $\operatorname{IC}\left(\mathfrak{g}_{1}, \mathcal{T}\left(\emptyset ; \mu^{1}, \mu^{2}\right)\right)$ and IC $\left(\mathfrak{g}_{1}, \mathcal{T}(\emptyset ; \mu, \mu)^{\omega}\right)$ are composition factors of the $\mathrm{IC}\left(\mathfrak{g}_{1}, \mathcal{L}_{\chi_{m}}\right)$. Hence (4.8) follows from (4.9).

Now let $\left(\nu ; \mu^{1}, \mu^{2}\right) \in \Sigma_{N}$ with $|\nu|=m>0$. Let

$\mathcal{K}\left(\rho_{\nu} \otimes V_{\mu^{1}, \mu^{2}}\right)$ denote the irreducible $L_{K^{-}}$equivariant local system on $\mathfrak{l}_{1}^{r s}$ associated to the irreducible representation of $\pi_{1}^{L_{K}}\left(\mathfrak{l}_{1}^{r s}\right)$ obtained as a pullback

$$
\text { of } \rho_{\nu} \otimes V_{\mu^{1}, \mu^{2}} \text { via the map } \pi_{1}^{L_{K}}\left(\mathfrak{l}_{1}^{r s}\right) \cong B_{m} \times \widetilde{B}_{N-2 m} \rightarrow S_{m} \times \widetilde{B}_{N-2 m} \text {. }
$$

By Proposition 3.2, we have that

$$
\mathrm{IC}\left(\mathfrak{g}_{1}^{m}, \mathcal{T}\left(\nu ; \mu^{1}, \mu^{2}\right)\right)=\operatorname{Ind}_{\mathfrak{r}_{1}^{m} \subset \mathfrak{p}_{1}^{m}}^{\mathfrak{g}_{1}} \operatorname{IC}\left(\mathfrak{l}_{1}, \mathcal{K}\left(\rho_{\nu} \otimes V_{\mu^{1}, \mu^{2}}\right)\right) .
$$

Since Fourier transform commutes with induction (see (3.9)), it suffices to show that the Fourier transform of IC $\left(\mathfrak{l}_{1}, \mathcal{K}\left(\rho_{\nu} \otimes V_{\mu^{1}, \mu^{2}}\right)\right)$ is supported on an $L_{K}$-nilpotent orbit in $\mathfrak{l}_{1}$. This follows from the classical Springer correspondence for $\mathfrak{g l}(m)$ and (4.8) applied to the symmetric pair $(\mathrm{SL}(N-2 m)$, $\mathrm{SO}(N-2 m))$ (see (3.10) $)$.

The proof for $\mathrm{IC}\left(\mathfrak{g}_{1}^{m}, \mathcal{T}(\nu ; \mu, \mu)^{\omega}\right), \operatorname{IC}\left(\mathfrak{g}_{1}^{n, \omega}, \mathcal{T}(\nu ; \emptyset, \emptyset)^{\omega}\right)$ proceeds in the same manner; in the latter case one uses the corresponding $\theta$-stable Levi and parabolic subgroups. We omit the details. 
4.2. More on induction. Let $\left(\nu ; \mu^{1}, \mu^{2}\right) \in \Sigma_{N}$. Assume that $|\nu|=m>0$. Let $L^{m} \subset P^{m}$ be as in 93 . Recall that $=L_{K}^{m} \cong \mathrm{GL}(m) \times \mathrm{SO}(N-2 m)$ and $\mathfrak{l}_{1}^{m} \cong \mathfrak{g l}(m) \oplus \mathfrak{s l}(N-2 m)_{1}$.

A nilpotent $L_{K}^{m}$-orbit in $\mathfrak{l}_{1}^{m}$ is given by a nilpotent orbit in $\mathfrak{g l}(m)$ and a nilpotent $\operatorname{SO}(N-$ $2 m)$-orbit in $\mathfrak{s l}(N-2 m)_{1}$. Thus the nilpotent $L_{K}^{m}$-orbits in $\mathfrak{l}_{1}^{m}$ are parametrized by $\mathcal{P}(m) \times$ $\mathcal{P}(N-2 m)$, with extra labels I and II for partitions in $\mathcal{P}(N-2 m)$ with all parts even. For $\alpha \in \mathcal{P}(m)$ and $\beta \in \mathcal{P}(N-2 m)$, we denote by $\mathcal{O}_{\alpha, \beta}$ (or $\left.\mathcal{O}_{\alpha, \beta}^{\omega}\right)$ the nilpotent $L_{K}^{m}$-orbit in $\mathfrak{l}_{1}^{m}$ given by the nilpotent orbit $\mathcal{O}_{\alpha}$ in $\mathfrak{g l}(m)$ and the nilpotent $\mathrm{SO}(N-2 m)$-orbit $\mathcal{O}_{\beta}$ (or $\left.\mathcal{O}_{\beta}^{\omega}\right)$ in $\mathfrak{s l}(N-2 m)_{1}$.

In the following we will omit the labels I and II with the understanding that everything should have corresponding labels, for example, $\mathcal{O}_{\lambda}^{\omega}=\operatorname{Ind}_{\mathfrak{r}_{1}^{m} \subset \mathfrak{p}_{1}^{m}}^{\mathfrak{g}_{1}} \mathcal{O}_{\alpha, \beta}^{\omega}$ etc.

Proposition 4.5. Let $\alpha \in \mathcal{P}(m)$ and $\beta \in \mathcal{P}(N-2 m)$. Let $\mathcal{O}_{\lambda}=\operatorname{Ind}_{\mathfrak{l}_{1}^{m} \subset \mathfrak{p}_{1}^{m}}^{\mathfrak{g}_{1}} \mathcal{O}_{\alpha, \beta}$, i.e., $\lambda_{i}=\beta_{i}+2 \alpha_{i}$. Assume that $u \in \mathcal{O}_{\alpha, \beta}$ and $v \in \mathcal{O}_{\lambda} \cap\left(u+\left(\mathfrak{n}_{P^{m}}\right)_{1}\right)$. We have a natural surjective map

$$
\psi: A_{K}(v) \rightarrow A_{L_{K}^{m}}(u) .
$$

Moreover, let $\mathbb{C} \otimes \mathcal{E}$ be an $L_{K}^{m}$-equivariant irreducible local system on $\mathcal{O}_{\alpha, \beta}$ and let $\tilde{\mathcal{E}}$ be the $K$-equivariant local system on $\mathcal{O}_{\lambda}$ obtained from $\mathbb{C} \otimes \mathcal{E}$ via the map $\psi$ above. Then $\operatorname{IC}\left(\mathcal{O}_{\lambda}, \tilde{\mathcal{E}}\right)$ is a direct summand of $\operatorname{Ind}_{\mathfrak{l}_{1}^{m} \subset \mathfrak{p}_{1}^{m}}^{\mathfrak{g}_{1}} \mathrm{IC}\left(\mathcal{O}_{\nu, \mu}, \mathbb{C} \otimes \mathcal{E}\right)$.

Corollary 4.6. If moreover $\left(\mathcal{O}_{\mu}, \mathcal{E}\right) \in \mathcal{A}_{N-2 m}$ is a pair such that $\mathfrak{F}\left(\mathrm{IC}\left(\mathcal{O}_{\mu}, \mathcal{E}\right)\right)$ has full support, then we have

$$
\operatorname{Ind}_{\mathfrak{r}_{1}^{m} \subset \mathfrak{p}_{1}^{m}}^{\mathfrak{g}_{1}} \mathrm{IC}\left(\mathcal{O}_{\nu, \mu}, \mathbb{C} \otimes \mathcal{E}\right) \cong \operatorname{IC}\left(\mathcal{O}_{\lambda}, \tilde{\mathcal{E}}\right)
$$

As before let us now write $L=L^{m}$ and $P=P^{m}$ etc. We begin the proof of the above proposition with the following lemma.

Lemma 4.7. The map

$$
\gamma: K \times^{P_{K}}\left(\overline{\mathcal{O}}_{\alpha, \beta}+\left(\mathfrak{n}_{P}\right)_{1}\right) \rightarrow \overline{\mathcal{O}}_{\lambda}
$$

is generically one-to-one.

Proof. Let $x_{0} \in \mathcal{O}_{\lambda}$. We can and will assume that $x_{0} \in \mathcal{O}_{\alpha, \beta}+\left(\mathfrak{n}_{P}\right)_{1}$. We show that $\gamma^{-1}\left(x_{0}\right)$ is a point. Assume that $\gamma(k, x)=x_{0}$. i.e. $\operatorname{Ad} k(x)=x_{0}$. Then $x \in \mathcal{O}_{\alpha, \beta}+\left(\mathfrak{n}_{P}\right)_{1}$. Let $\widetilde{\mathcal{O}}_{\lambda}$ (resp. $\widetilde{\mathcal{O}}_{\alpha, \beta}$ ) be the (unique) $G$-orbit (resp. L-orbit) in $\mathfrak{g}$ (resp. $\mathfrak{l}$ ) that contains $\mathcal{O}_{\lambda}$ (resp. $\left.\mathcal{O}_{\alpha, \beta}\right)$. We have that

$$
\widetilde{\mathcal{O}}_{\lambda}=\operatorname{Ind}_{\mathfrak{l}}^{\mathfrak{g}} \widetilde{\mathcal{O}}_{\alpha, \beta}
$$

in the notation of Lusztig and Spaltenstein [LS]. By [LS, Theorem 1.3], we have $Z_{G}^{0}\left(x_{0}\right) \subset$ $P$. In fact, we have that $Z_{G}\left(x_{0}\right) \subset P$. This can be seen by enlarging the group $G$ to $G L(N)$ and using the fact that $Z_{G L(N)}\left(x_{0}\right)$ is connected. Thus $Z_{K}\left(x_{0}\right) \subset P_{K}$. Furthermore, $\widetilde{\mathcal{O}}_{\lambda} \cap\left(\widetilde{\mathcal{O}}_{\alpha, \beta}+\mathfrak{n}_{P}\right)$ is a single orbit under $P$. Thus there exists $p \in P$ such that $\operatorname{Ad} p(x)=x_{0}$. It follows that $k^{-1} p \in Z_{G}\left(x_{0}\right) \subset P$. Thus $k \in P \cap K=P_{K}$. Now we have that $(k, x)=$ $(1, \operatorname{Ad} k(x))=\left(1, x_{0}\right)$. 
Proof of Proposition 4.5. Note that the proof of the above lemma shows that $Z_{G}(v)=Z_{P}(v)$. We have $Z_{P}(v) \subset Z_{L}(u) U_{P}$. Thus $Z_{K}(v)=Z_{P_{K}}(v) \subset Z_{L_{K}}(u)\left(U_{P} \cap K\right)$. It follows that we have a natural projection map

$$
Z_{K}(v) / Z_{K}^{0}(v)=Z_{P_{K}}(v) / Z_{P_{K}}^{0}(v) \rightarrow Z_{L_{K}}(u) / Z_{L_{K}}^{0}(u) .
$$

We show that this gives us the desired map $\psi$. Following [LS], we have that $Z_{L_{K}}(u)\left(U_{P} \cap K\right)$ has a dense orbit, i.e. the orbit of $v$, in the irreducible variety $u+\left(\mathfrak{n}_{P}\right)_{1}$. Thus $Z_{P_{K}}(v)=Z_{K}(v)$ meets all the irreducible components of $Z_{L_{K}}(u)\left(U_{P} \cap K\right)$, which implies that $\psi$ is surjective.

It is easy to see that

$$
\operatorname{supp}\left(\operatorname{Ind}_{\mathfrak{l}_{1} \subset \mathfrak{p}_{1}}^{\mathfrak{g}_{1}} \operatorname{IC}\left(\mathcal{O}_{\alpha, \beta}, \mathbb{C} \otimes \mathcal{E}\right)\right)=\overline{\mathcal{O}}_{\lambda} .
$$

The proposition follows from the definition of parabolic induction functor and Lemma 4.7.

Remark 4.1. The proof of Lemma 4.7 and the existence and surjectivity of the map $\psi$ works for any $\theta$-stable Levi contained in a $\theta$-stable parabolic subgroup.

Proof of Corollary 4.6. Note that the assumption implies that $\mathfrak{F}\left(\operatorname{IC}\left(\mathcal{O}_{\alpha, \beta}, \mathbb{C} \otimes \mathcal{E}\right)\right)$ has full support, i.e. $\operatorname{IC}\left(\mathcal{O}_{\alpha, \beta}, \mathbb{C} \otimes \mathcal{E}\right)=\operatorname{IC}\left(\mathfrak{l}_{1}, \mathcal{G}\right)$ for some irreducible $L_{K}$-equivariant local system $\mathcal{G}$ on $\mathfrak{l}_{1}^{r s}$. We have that

$$
\mathfrak{F}\left(\operatorname{Ind}_{\mathfrak{l}_{1} \subset \mathfrak{p}_{1}}^{\mathfrak{g}_{1}} \operatorname{IC}\left(\mathcal{O}_{\alpha, \beta}, \mathbb{C} \otimes \mathcal{E}\right)\right)=\operatorname{Ind}_{\mathfrak{l}_{1} \subset \mathfrak{p}_{1}}^{\mathfrak{g}_{1}} \mathfrak{F}\left(\operatorname{IC}\left(\mathcal{O}_{\alpha, \beta}, \mathbb{C} \otimes \mathcal{E}\right)\right)=\operatorname{Ind}_{\mathfrak{l}_{1} \subset \mathfrak{p}_{1}}^{\mathfrak{g}_{1}} \operatorname{IC}\left(\mathfrak{l}_{1}, \mathcal{G}\right) .
$$

It suffices to show that $\operatorname{Ind}_{\mathfrak{l}_{1} \subset \mathfrak{p}_{1}}^{\mathfrak{g}_{1}} \operatorname{IC}\left(\mathfrak{l}_{1}, \mathcal{G}\right)$ is irreducible. This follows from the definition of the induction functor and Proposition 3.1 .

Corollary 4.8. The Fourier transform of a nilpotent orbital complex $\operatorname{IC}(\mathcal{O}, \mathcal{E}) \in \mathcal{A}_{N}$ has full support, i.e., $\operatorname{supp} \mathfrak{F}(\mathrm{IC}(\mathcal{O}, \mathcal{E}))=\mathfrak{g}_{1}$, if and only if it is not of the form $\operatorname{Ind}_{\mathfrak{l}_{1} \subset \mathfrak{p}_{1}} \operatorname{IC}\left(\mathcal{O}^{\prime}, \mathcal{E}^{\prime}\right)$ where supp $\mathfrak{F}\left(\mathrm{IC}\left(\mathcal{O}^{\prime}, \mathcal{E}^{\prime}\right)\right)=\mathfrak{l}_{1}$, and $L \subset P$ is a pair chosen as in $\$$.

Proof. The only if part follows from the facts that Fourier transform commutes with parabolic induction and that supp $\operatorname{Ind}_{\mathfrak{l}_{1} \subset \mathfrak{p}_{1}}^{\mathfrak{g}_{1}} A \subsetneq \mathfrak{g}_{1}$. The if part follows from (4.10), (4.6) and Theorem 4.1.

Corollary 4.9. Let $\lambda=\left(\lambda_{1} \geq \lambda_{2} \geq \cdots\right) \in \mathcal{P}(N)$.

(1) If $\lambda_{i}-\lambda_{i+1} \geq 3$ for some $i$, then $\operatorname{supp} \mathfrak{F}\left(\operatorname{IC}\left(\mathcal{O}_{\lambda}, \mathcal{E}\right)\right) \neq \mathfrak{g}_{1}$ for any $K$-equivariant local system $\mathcal{E}$ on $\mathcal{O}_{\lambda}$. The same holds for $\mathcal{O}_{\lambda}^{\omega}$ if $\lambda$ has only even parts.

(2) Suppose that $\lambda_{i}-\lambda_{i+1} \leq 2$ for all $i$. Let $f_{\lambda}$ be the number of different sizes of parts of $\lambda$, and $g_{\lambda}$ the number of $i$ 's such that $\lambda_{i}-\lambda_{i+1}=2$.

(a) If at least one part of $\lambda$ is odd, then there are $2^{f_{\lambda}-1-g_{\lambda}}$ irreducible $K$-equivariant local systems $\mathcal{E}$ on $\mathcal{O}_{\lambda}$ such that $\operatorname{supp} \mathfrak{F}\left(\operatorname{IC}\left(\mathcal{O}_{\lambda}, \mathcal{E}\right)\right)=\mathfrak{g}_{1}$. 
(b) If all parts of $\lambda$ are even, then there is exactly one irreducible $K$-equivariant local system $\mathcal{E}^{\omega}$ on each orbit $\mathcal{O}_{\lambda}^{\omega}, \omega=\mathrm{I}, \mathrm{II}$, such that $\operatorname{supp} \mathfrak{F}\left(\mathrm{IC}\left(\mathcal{O}_{\lambda}^{\omega}, \mathcal{E}^{\omega}\right)\right)=\mathfrak{g}_{1}$.

In particular, if $\lambda_{i}-\lambda_{i+1} \leq 1$ for all $i$, then $\operatorname{supp} \mathfrak{F}\left(\operatorname{IC}\left(\mathcal{O}_{\lambda}, \mathcal{E}\right)\right)=\mathfrak{g}_{1}$ for any $K$ equivariant local system $\mathcal{E}$ on $\mathcal{O}_{\lambda}$.

Proof. (1) Assume that $\lambda_{i_{0}}-\lambda_{i_{0}+1} \geq 3$. Let $m=i_{0}, \alpha=1^{i_{0}}, \beta=\left(\lambda_{1}-2, \ldots, \lambda_{i_{0}}-\right.$ $\left.2, \lambda_{i_{0}+1}, \ldots\right)$. Then $\mathcal{O}_{\lambda}=\operatorname{Ind}_{\mathfrak{l}_{1}^{m} \subset \mathfrak{p}_{1}^{m}}^{\mathfrak{g}_{1}} \mathcal{O}_{\alpha, \beta}$. Let $u \in \mathcal{O}_{\alpha, \beta}$ and $v \in \mathcal{O}_{\lambda} \cap\left(u+\left(\mathfrak{n}_{P^{m}}\right)_{1}\right)$. Note that $A_{K}(v) \cong A_{L_{K}^{m}}(u)$. It then follows from Proposition 4.5 that for each irreducible $K$ equivariant local system $\mathcal{E}$ on $\mathcal{O}_{\lambda}, \operatorname{IC}\left(\mathcal{O}_{\lambda}, \mathcal{E}\right)$ is a direct summand of $\operatorname{Ind}_{\mathfrak{l}_{1}^{m} \subset \mathfrak{p}_{1}^{m}}^{\mathfrak{g}_{1}} \operatorname{IC}\left(\mathcal{O}_{\alpha, \beta}, \mathcal{E}_{0}\right)$ for some irreducible $L_{K}$-equivariant local system $\mathcal{E}_{0}$ on $\mathcal{O}_{\alpha, \beta}$. As before, this shows that $\mathfrak{F}\left(\mathrm{IC}\left(\mathcal{O}_{\lambda}, \mathcal{E}\right)\right)$ has smaller support.

In the case when $\lambda$ has only even parts, we let $\mathcal{O}_{\lambda}^{\omega}=\operatorname{Ind}_{\mathfrak{r}_{1}^{m} \subset \mathfrak{p}_{1}^{m}}^{\mathfrak{g}_{1}} \mathcal{O}_{\alpha, \beta}^{\omega}$, if $m<N / 2$, and we let $\mathcal{O}_{\lambda}^{\omega}=\operatorname{Ind}_{\mathfrak{l}_{1}^{n, \omega} \subset \mathfrak{p}_{1}^{n, \omega}}^{\mathfrak{g}_{1}} \mathcal{O}_{\alpha, \beta}$, if $m=N / 2=n$, where $\omega=\mathrm{I}$, II. The proof for $\mathcal{O}_{\lambda}^{\omega}$ then proceeds in the same way.

(2) We argue by induction on $g_{\lambda}$. If $g_{\lambda}=0$, then (2) follows from (4.11) and Corollary 4.8, Assume by induction hypothesis that (2) holds for all $\mu$ with $g_{\mu}<g_{\lambda}$.

Assume first that $\lambda$ has at least one odd part. Suppose that $i_{1}, \ldots, i_{k}$ are such that $\lambda_{i_{j}}-\lambda_{i_{j}+1}=2$, where $k=g_{\lambda}$.

Let $a=\left(a_{1} \geq a_{2} \geq \cdots \geq a_{k} \geq 0\right)$ be a partition such that $a \neq \emptyset, a_{k} \leq 1$, and $a_{l} \leq a_{l+1}-1$. Note that the number of such partitions is $2^{k}-1$. Consider a partition $\mu(a)$ such that $\mu_{l}=\lambda_{l}-2 a_{j}$ for $l \in\left[i_{j-1}+1, i_{j}\right]$. Then $\mu(a)$ satisfies that $\mu(a)_{i}-\mu(a)_{i+1} \leq 2$ and $g_{\mu(a)}<g_{\lambda}$. Moreover, $\mu$ has at least one odd part, and $f_{\lambda}-g_{\lambda}=f_{\mu(a)}-g_{\mu(a)}$. Let $m=\sum_{j=1}^{k} i_{j}$. We have that

$$
\operatorname{Ind}_{\mathfrak{r}_{1}^{m} \subset \mathfrak{p}_{1}^{m}}^{\mathfrak{g}_{1}} \mathcal{O}_{a, \mu(a)}=\mathcal{O}_{\lambda}
$$

By induction hypothesis, there are $2^{f_{\lambda}-g_{\lambda}-1}$ irreducible $K$-equivariant local systems $\mathcal{E}$ on $\mathcal{O}_{a, \mu(a)}$ such that $\mathfrak{F}\left(\mathrm{IC}\left(\mathcal{O}_{a, \mu(a)}, \mathcal{E}\right)\right.$ has full support. By Corollary 4.6, we have that

$$
\operatorname{Ind}_{\mathfrak{l}_{1}^{m} \subset \mathfrak{p}_{1}^{m}}^{\mathfrak{g}_{1}} \operatorname{IC}\left(\mathcal{O}_{a, \mu(a)}, \mathcal{E}\right)=\operatorname{IC}\left(\mathcal{O}_{\lambda}, \tilde{\mathcal{E}}\right)
$$

This gives rise to $\left(2^{k}-1\right) \cdot 2^{f_{\lambda}-g_{\lambda}-1}=2^{f_{\lambda}-1}-2^{f_{\lambda}-g_{\lambda}-1}$ irreducible $K$-equivariant local systems $\tilde{\mathcal{E}}$ on $\mathcal{O}_{\lambda}$ such that $\mathfrak{F}\left(\operatorname{IC}\left(\mathcal{O}_{\lambda}, \tilde{\mathcal{E}}\right)\right.$ has smaller support (with $a$ varying).

The case when all parts of $\lambda$ even can be argued in the same way. Note that in this case $g_{\lambda}=f_{\lambda}$.

Let us write $m_{\lambda}$ (resp. $m_{\lambda}^{\omega}, \omega=\mathrm{I}, \mathrm{II}$ ) for the number of irreducible $K$-equivariant local systems $\tilde{\mathcal{E}}$ on $\mathcal{O}_{\lambda}\left(\operatorname{resp} . \mathcal{O}_{\lambda}^{\omega}\right)$ such that $\mathfrak{F}\left(\operatorname{IC}\left(\mathcal{O}_{\lambda}, \tilde{\mathcal{E}}\right)\left(\operatorname{resp} . \mathfrak{F}\left(\operatorname{IC}\left(\mathcal{O}_{\lambda}^{\omega}, \tilde{\mathcal{E}}\right)\right)\right.\right.$ has full support when at least one part of $\lambda$ is odd (resp. when all parts of $\lambda$ are even). 
We conclude from the discussion above that

$$
\begin{gathered}
m_{\lambda} \leq 2^{f_{\lambda}-g_{\lambda}-1} \text { if } \lambda \text { has at least one odd part, } \\
\text { resp. } m_{\lambda}^{\omega} \leq 1 \text { if all parts of } \lambda \text { are even. }
\end{gathered}
$$

Theorem 4.1 implies that the number of pairs $\operatorname{IC}(\mathcal{O}, \mathcal{E}) \in \mathcal{A}_{N}$ such that $\operatorname{supp} \mathfrak{F}(\operatorname{IC}(\mathcal{O}, \mathcal{E}))=\mathfrak{g}_{1}$ is $d(n)$ (see (4.1)), when $N=2 n+1$, and $e(n)$ (see (4.2)), when $N=2 n$. In view of (4.12) and claim (1) of the corollary, it suffices to show that

$$
\sum_{\substack{\lambda \in \mathcal{P}(2 n+1) \\ \lambda_{i}-\lambda_{i+1} \leq 2}} 2^{f_{\lambda}-g_{\lambda}-1}=d(n), \sum_{\substack{\lambda \in \mathcal{P}(2 n), \lambda_{i}-\lambda_{i+1} \leq 2, \\ \text { not all parts of } \lambda \text { even }}} 2^{f_{\lambda}-g_{\lambda}-1}+2 q(n)=e(n) .
$$

This can be seen as follows. Note that when $N$ is even, the number of orbits of the form $\mathcal{O}_{\lambda}^{\omega}$, where all parts of $\lambda$ are even and $\lambda_{i}-\lambda_{i+1} \leq 2$, is $2 q(n)$. We know that

$$
\begin{gathered}
d(n)=\text { Coefficient of } x^{2 n+1} \text { in } \frac{1}{2} \prod_{s \geq 1}\left(1+x^{s}\right)^{2} . \\
e(n)=\frac{3}{2} q(n)+\text { Coefficient of } x^{2 n} \text { in } \frac{1}{2} \prod_{s \geq 1}\left(1+x^{s}\right)^{2} .
\end{gathered}
$$

A partition $\lambda$ satisfies that $\lambda_{i}-\lambda_{i+1} \leq 2$ if and only if each part of the transpose partition $\lambda^{\prime}$ has multiplicity at most 2 . We have $f_{\lambda}=f_{\lambda^{\prime}}$ and $g_{\lambda}$ equals the number of parts in $\lambda^{\prime}$ with multiplicity 2 . It is easy to see that each $\lambda^{\prime}$ whose parts have multiplicity at most 2 appears in $\prod_{s \geq 1}\left(1+x^{s}\right)^{2}$ exactly $2^{f_{\lambda}-g_{\lambda}}$ times. Hence (4.13) follows.

Remark 4.2. In [CVX1, Conjecture 1.2], we conjectured that one can obtain all nilpotent orbital complexes by induction from those of smaller groups whose Fourier transforms have full support. This conjecture follows from Corollary 4.8.

\section{Cohomology of Hessenberg varieties}

Hessenberg varieties, defined generally in GKM], arise naturally in our setting (for details, see CVX2]). In particular, they arise as fibers of maps $\pi$ and $\check{\pi}$ in the following diagram

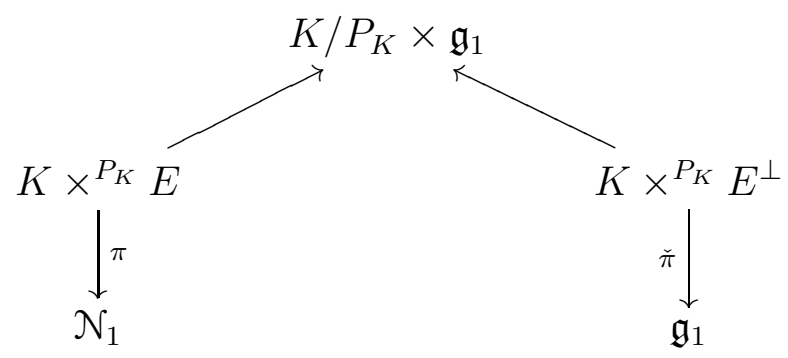

where $P_{K}$ is a parabolic subgroup of $K, E$ is a $P_{K}$-stable subspace of $\mathfrak{g}_{1}$ consisting of nilpotent elements, and $E^{\perp}$ is the orthogonal complement of $E$ in $\mathfrak{g}_{1}$ via a $K$-invariant non-degenerate form on $\mathfrak{g}$. The generic fibers of maps $\check{\pi}$ are Hessenberg varieties. 
In this section we discuss an application of our result to cohomology of Hessenberg varieties. Let us fix $s \in \mathfrak{g}_{1}^{r s}$ and consider the corresponding Hessenberg variety

$$
\text { Hess }:=\check{\pi}^{-1}(s)=\left\{g P_{K} \in K / P_{K} \mid g^{-1} s g \in E^{\perp}\right\} .
$$

The centralizer $Z_{K}(s)$ acts naturally on Hess and it induces an action of the component group $\pi_{0}\left(Z_{K}(s)\right) \cong I_{N}$ on the cohomology groups $H^{*}($ Hess, $\mathbb{C})$. Let

$$
\mathrm{H}^{*}(\text { Hess }, \mathbb{C})=\bigoplus_{\chi \in I_{N}^{\vee}} \mathrm{H}^{*}(\text { Hess }, \mathbb{C})_{\chi}
$$

be the eigenspace decomposition with respect to the action of $I_{N}$.

Definition 5.1. The stable part $\mathrm{H}^{*}(\text { Hess, } \mathbb{C})_{\text {st }}$ of $\mathrm{H}^{*}(\mathrm{Hess}, \mathbb{C})$ is the direct summand $\mathrm{H}^{*}(\text { Hess }, \mathbb{C})_{\chi_{\text {triv }}}$ where $\chi_{\text {triv }} \in I_{N}^{\vee}$ is the trivial character.

For simplicity we now assume $\check{\pi}$ is onto. In this case $\check{\pi}$ is smooth over $\mathfrak{g}_{1}^{s}$ (e.g. see CVX2, Lemma 2.1]) and the equivariant fundamental group $\pi_{1}^{K}\left(\mathfrak{g}_{1}^{r s}, s\right) \cong I_{N} \rtimes B_{N}$ acts on $H^{*}($ Hess, $\mathbb{C})$ by the monodromy action. Recall that for $\chi \in I_{N}^{\vee}, B_{\chi}$ stands for the stabilizer of $\chi$ in $B_{N}$. Clearly, each summand $H^{*}(\text { Hess, } \mathbb{C})_{\chi}$ is stable under the action of $B_{\chi}$. Let $\chi_{m} \in I_{N}^{\vee}, B_{\chi_{m}}$, and $B_{m, N-m}$ be as in 92.3 . Assume that $\chi$ is in the $B_{N^{-o r b i t}}$ of $\chi_{m}$. Then for any $b \in B_{N}$ with $b \cdot \chi=\chi_{m}$ we have an isomorphism $\iota_{b}: B_{\chi} \cong B_{\chi_{m}}, u \rightarrow b u b^{-1}$. Note that $\chi_{\text {triv }}=\chi_{0}$ and $B_{\chi_{m}}=B_{m, N-m}$ except when $N$ is even and $m=N / 2$. In that case, $B_{m, N-m}$ is an index two subgroup of $B_{\chi_{m}}$.

Recall the algebra $\mathcal{H}_{\chi_{m},-1}=\mathcal{H}_{m,-1} \times \mathcal{H}_{N-m,-1}$ and their representations $D_{\mu^{1}} \otimes D_{\mu^{2}}$ introduced in $\$ 2.3$. Each $\mathcal{H}_{\chi_{m},-1}$ is a quotient of the group algebra $\mathbb{C}\left[B_{m, N-m}\right]$ and $\mathcal{H}_{\chi_{0},-1}=$ $\mathcal{H}_{\chi_{\text {triv }},-1}=\mathcal{H}_{N,-1}$ is the Hecke algebra of $S_{N}$ at $q=-1$.

Theorem 5.1. (1) Let $\chi_{m} \in I_{N}^{\vee}$ be the representatives of $B_{N}$-orbtis in \$2.3. To every $\chi \in$ $I_{N}^{\vee}$ in the orbit of $\chi_{m}$ and an element $b \in B_{N}$ satisfying $b(\chi)=\chi_{m}$, the monodromy action of $b$ on $\mathrm{H}^{*}($ Hess, $\mathbb{C})$ induces an isomorphism $\mathrm{H}^{*}(\text { Hess, } \mathbb{C})_{\chi} \cong \mathrm{H}^{*}(\text { Hess, } \mathbb{C})_{\chi_{m}}$ compatible with the actions of $B_{\chi} \stackrel{\iota_{b}}{\cong} B_{\chi_{m}}$ on both sides.

(2) The action of $\mathbb{C}\left[B_{m, N-m}\right]$ on $\mathrm{H}^{*}(\text { Hess, } \mathbb{C})_{\chi_{m}}$ factors through the algebra $\mathcal{H}_{\chi_{m},-1}$ and the resulting representation is a direct sum of $D_{\mu^{1}} \otimes D_{\mu^{2}}, \mu^{1} \in \mathcal{P}_{2}(m), \mu^{2} \in \mathcal{P}_{2}(N-m)$. In particular, the stable part $\mathrm{H}^{*}(\mathrm{Hess}, \mathbb{C})_{\mathrm{st}}$ is generated by irreducible representations of the Hecke algebra of $S_{N}$ at $q=-1$.

Proof. Part (1) is clear. To prove part (2) we proceed as follows. By the decomposition theorem $\pi_{*} \mathbb{C}$ is a direct sum of shifts of nilpotent orbital complexes. Since $\mathfrak{F}\left(\pi_{*} \mathbb{C}\right) \cong \check{\pi}_{*} \mathbb{C}$ (up to shift), Theorem 4.1 implies that a generic stalk of $\check{\pi}_{*} \mathbb{C}$, which is isomorphic to $H^{*}($ Hess, $\mathbb{C})$, is a direct sum of the local systems $V_{\mu^{1}, \mu^{2}}=\operatorname{Ind}_{\mathbb{C}\left[B_{m, N-m}\right]}^{\mathbb{C}\left[B_{N}\right]} D_{\mu_{1}} \otimes D_{\mu_{2}}$ introduced in (2.6) . Since $I_{N}$ acts on $V_{\mu^{1}, \mu^{2}}$ by the formula $a .(b \otimes v)=\left(\left(b \cdot \chi_{m}\right)(a)\right)(b \otimes v)$ for $a \in I_{N}, b \in B_{N}$ and $v \in D_{\mu_{1}} \otimes D_{\mu_{2}}$, we have $\left(V_{\mu^{1}, \mu^{2}}\right)_{\chi} \cong D_{\mu_{1}} \otimes D_{\mu_{2}}$. The theorem follows. 
Example 5.1. Let $C$ be the hyper-elliptic curve with affine equation $y^{2}=\prod_{j=1}^{N}\left(x-a_{j}\right)$ (here $a_{i} \neq a_{j}$ for $\left.i \neq j\right)$. Assume $N=2 n+2$ is even. Then according to CVX3, Section 2.3] the Jacobian $\operatorname{Jac}(C)$ is an example of Hessenberg variety and the monodromy action of $\pi_{1}\left(\mathfrak{g}_{1}^{r s}, s\right)$ factors through $B_{N}$, that is, $\mathrm{H}^{*}(\operatorname{Jac}(C), \mathbb{C})=\mathrm{H}^{*}(\operatorname{Jac}(C), \mathbb{C})_{\text {st }}$. Let $\mu_{k}=(N-k, k) \in \mathcal{P}_{2}(N)$ and $D_{\mu_{k}}$ be the corresponding representation of $\mathcal{H}_{N,-1}$. Using [A], one can check that the induced action of the group algebra $\mathbb{C}\left[B_{N}\right]$ on $\mathrm{H}^{i}(\operatorname{Jac}(C), \mathbb{C})$ factors through $\mathcal{H}_{N,-1}$ and for $i \leq n$ the resulting representation of $\mathcal{H}_{N,-1}$ is isomorphic to

$$
\mathrm{H}^{i}(\operatorname{Jac}(C), \mathbb{C}) \cong \bigoplus_{j=0}^{[i / 2]} D_{\mu_{i-2 j}}
$$

with the primitive part $\mathrm{H}^{i}(\operatorname{Jac}(C), \mathbb{C})_{\text {prim }} \cong D_{\mu_{i}}$.

Remark 5.2. It would be nice to have an explicit decomposition of $H^{*}(\text { Hess, } \mathbb{C})_{\chi_{m}}$ into irreducible representations of $\mathcal{H}_{\chi_{m},-1}$. For this one needs finer information for the bijection in Theorem 4.1 (see Section 7). In CVX1, CVX3, we establish an explicit bijection for certain nilpotent orbital complexes and together with other results, we work out an explicit decomposition in the case when the Hessenberg varieties are isomorphic to Fano varieties of $k$-planes in smooth complete intersections of two quadrics in projective space.

\section{Representations of $\mathcal{H}_{N,-1}$}

In this section we show that all irreducible representations of the Hecke algebra $\mathcal{H}_{N,-1}$ come from geometry. Indeed they all appear in intersection cohomology of a Hessenberg variety with coefficient in a local system. In particular, this shows that all irreducible representations of $\mathcal{H}_{N,-1}$ carry a Hodge structure. In particular, the irreducible representations of $\mathcal{H}_{N,-1}$ can be viewed as variations of Hodge structure.

Let $\mathcal{O}$ be a nilpotent $K$-orbit on $\mathfrak{g}_{1}$ and $\mathcal{L}$ an irreducible $K$-equivariant local system on $\mathcal{O}$. We call $(\mathcal{O}, \mathcal{L})$ a nilpotent pair. Following $[\mathrm{LY}]$, we associate to each nilpotent pair $(\mathcal{O}, \mathcal{L})$ two families of Hessenberg varieties $\operatorname{Hess}_{\mathcal{L}, \pm 1} \rightarrow \mathfrak{g}_{1}$ together with local systems $\hat{\mathcal{L}}_{ \pm 1}$ on open subsets $\operatorname{Hess}_{\mathcal{L}, \pm 1} \subset \operatorname{Hess}_{\mathcal{L}, \pm 1}$.

Let $x \in \mathfrak{g}_{1}$ be a nilpotent element in $\mathcal{O}$. Choose a normal $\mathrm{sl}_{2}$-triple $\{x, h, y\}$ and let

$$
\mathfrak{g}(i)=\{v \in \mathfrak{g} \mid[h, v]=i v\}, \mathfrak{g}_{0}(i)=\mathfrak{g}(i) \cap \mathfrak{g}_{0}, \text { and } \mathfrak{g}_{1}(i)=\mathfrak{g}(i) \cap \mathfrak{g}_{1}
$$

For any $N \in \mathbb{Z}$ we write $\underline{N} \in\{0,1\}$ for its image in $\mathbb{Z} / 2 \mathbb{Z}$. Define

$$
\mathfrak{p}_{N}^{x}=\bigoplus_{k \geq 2 N} \mathfrak{g}_{\underline{N}}(k), \mathfrak{l}_{N}^{x}=\mathfrak{g}_{\underline{N}}(2 N), \text { and } \mathfrak{l}^{x}=\bigoplus_{N \in \mathbb{Z}} \mathfrak{l}_{N}^{x}
$$

One can check that $\mathfrak{l}^{x} \subset \mathfrak{g}$ is a graded Lie subalgebra of $\mathfrak{g}$ and $x \in \mathfrak{l}_{1}^{x}=\mathfrak{g}_{1}(2)$. Let $L_{0}^{x} \subset K$ be the reductive subgroup with Lie algebra $\mathfrak{l}_{0}^{x}=\mathfrak{g}_{0}(0)$. By [LY, 2.9(c)], the restriction

$$
\mathcal{L}_{1}^{\prime}:=\left.\mathcal{L}\right|_{\mathfrak{r}_{1}^{x}}
$$

is an irreducible $L_{0}^{x}$-equivariant local system on the unique open $L_{0}^{x}$-orbit $\mathfrak{l}_{1}^{x}$ on $\mathfrak{l}_{1}^{x}$. 
According to [L1], there exists a graded parabolic subalgebra $\mathfrak{q}=\bigoplus_{N \in \mathbb{Z}} \mathfrak{q}_{N}$ of $\mathfrak{l}^{x}$, a Levi subalgebra $\mathfrak{m}=\bigoplus_{N \in \mathbb{Z}} \mathfrak{m}_{N}$ of $\mathfrak{q}$, and a cuspidal local system $\mathcal{L}_{1}$ on the open $M_{0}$-orbit $\mathfrak{m}_{1}$ of $\mathfrak{m}_{1}$ (here $M_{0}$ is the reductive subgroup of $L_{0}^{x}$ with Lie algebra $\mathfrak{m}_{0}$ ) such that

some shift of the IC-complex $\operatorname{IC}\left(\mathfrak{l}_{1}^{x}, \mathcal{L}_{1}^{\prime}\right)$ is a direct summand of $\operatorname{Ind}_{\mathfrak{m}_{1} \subset \mathfrak{q}_{1}}^{\mathfrak{l}_{1}^{x}} \operatorname{IC}\left(\mathfrak{m}_{1}, \mathcal{L}_{1}\right)$.

In addition, we have

$$
\mathfrak{F}\left(\mathrm{IC}\left(\mathfrak{m}_{1}, \mathcal{L}_{1}\right)\right) \cong \mathrm{IC}\left(\mathfrak{m}_{-1}, \mathcal{L}_{-1}\right)
$$

where $\mathcal{L}_{-1}$ is a cuspical local system on the unique open orbit $\stackrel{\circ}{\mathfrak{m}}_{-1} \subset \mathfrak{m}_{-1}$.

Define $\hat{\mathfrak{q}}_{N}$ to be the pre-image of $\mathfrak{q}_{N}$ under the projection map $\mathfrak{p}_{N}^{x} \rightarrow \mathfrak{l}_{N}^{x}$. Let $Q_{K} \subset K$ be

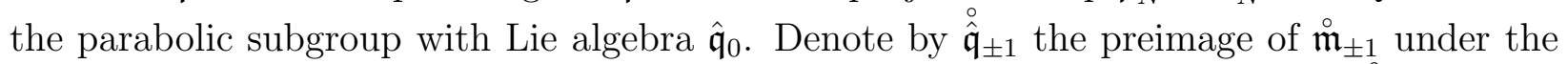
projection map $\hat{\mathfrak{q}}_{ \pm 1} \rightarrow \mathfrak{q}_{ \pm 1} \rightarrow \mathfrak{m}_{ \pm 1}$. The group $Q_{K}$ acts naturally on $\hat{\mathfrak{q}}_{ \pm 1}$ and $\hat{\hat{\mathfrak{q}}}_{ \pm 1}$ and we define

Let

$$
\operatorname{Hess}_{\mathcal{L}, \pm 1}:=K \times^{Q_{K}} \hat{\mathfrak{q}}_{ \pm 1}, \operatorname{Hess}_{\mathcal{L}, \pm 1}^{\circ}:=K \times^{Q_{K}} \stackrel{\circ}{\hat{\mathfrak{q}}}_{ \pm 1}
$$

$$
\pi_{\mathcal{L}, \pm 1}: \operatorname{Hess}_{\mathcal{L}, \pm 1} \rightarrow \mathfrak{g}_{1},(x, v) \rightarrow x v x^{-1}
$$

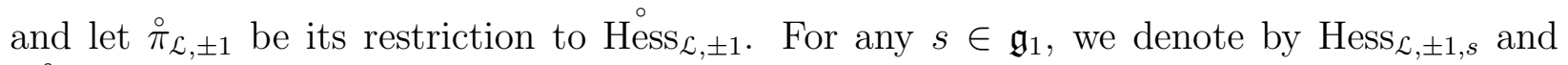

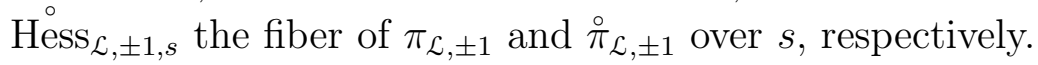

There are natural maps

$$
h_{\mathcal{L}, \pm 1}: \operatorname{Hess}_{\mathcal{L}, \pm 1} \rightarrow\left[\mathfrak{m}_{ \pm 1} / M_{0}\right], \quad \stackrel{\circ}{h}_{\mathcal{L}, \pm 1}: \operatorname{Hess}_{\mathcal{L}, \pm 1} \rightarrow\left[\stackrel{\circ}{\mathfrak{m}}_{ \pm 1} / M_{0}\right]
$$

sending $(k, v) \in \operatorname{Hess}_{\mathcal{L}, \pm 1}=K \times \times^{Q_{K}} \hat{\mathfrak{q}}_{ \pm 1}$ to $\bar{v}$, the image of $v \in \hat{\mathfrak{q}}_{ \pm 1}$ under the map $\hat{\mathfrak{q}}_{ \pm 1} \rightarrow$ $\mathfrak{m}_{ \pm 1} \rightarrow\left[\mathfrak{m}_{ \pm 1} / M_{0}\right]$. We define the following local system

$$
\hat{\mathcal{L}}_{ \pm 1}:=\left(\stackrel{\circ}{h}_{\mathcal{L}, \pm 1}\right)^{*} \mathcal{L}_{ \pm 1}
$$

on $\operatorname{Hess}_{\mathcal{L}, \pm 1}$. Here we view the $M_{0}$-local systems $\mathcal{L}_{ \pm 1}$ as sheaves on $\left[\mathfrak{\mathfrak { m }}_{ \pm 1} / M_{0}\right]$.

Example 6.1. Consider the nilpotent pair $\left(\mathcal{O}, \mathcal{L}=\mathcal{L}_{\text {triv }}\right)$ where $\mathcal{L}_{\text {triv }}$ is the trivial local system on $\mathcal{O}$. Using [L1, Proposition 7.3] one can check that in this case $\mathfrak{q}=\oplus_{N \in \mathbb{Z}} \mathfrak{q}_{N}$ is a Borel subalgebra of $\mathfrak{l}^{x}$ and $\mathfrak{m}=\oplus_{N \in \mathbb{Z}} \mathfrak{m}_{N}$ is a Cartan subalgebra. Moreover the grading on $\mathfrak{m}$ is concentrated in degree zero, i.e., $\mathfrak{m}=\mathfrak{m}_{0}$, and the cuspidal local system $\mathcal{L}_{ \pm 1}$ is the skyscraper sheaf supported on $\mathfrak{m}_{ \pm 1}=\{0\}$. It follows that in this case $\operatorname{Hess}_{\mathcal{L}_{\text {triv }}, \pm 1}=\operatorname{Hess}_{\mathcal{L}_{\text {triv }}, \pm 1}$ and $\hat{\mathcal{L}}_{ \pm 1}$ is the constant local system.

In [LY, $\S 7]$, the authors prove the following:

$$
\left(\pi_{\mathcal{L},-1}\right)_{*} \operatorname{IC}\left(\operatorname{Hess}_{\mathcal{L},-1}, \hat{\mathcal{L}}_{-1}\right) \text { is the Fourier transform of }\left(\pi_{\mathcal{L}, 1}\right)_{*} \operatorname{IC}\left(\operatorname{Hess}_{\mathcal{L}, 1}, \hat{\mathcal{L}}_{1}\right) .
$$

Some shift of $\operatorname{IC}(\overline{\mathcal{O}}, \mathcal{L})$ (resp. the Fourier transform of $\operatorname{IC}(\overline{\mathcal{O}}, \mathcal{L}))$ appears in $\left(\pi_{\mathcal{L}, 1}\right)_{*} \operatorname{IC}\left(\operatorname{Hess}_{\mathcal{L}, 1}, \hat{\mathcal{L}}_{1}\right)$ (resp. $\left.\left(\pi_{\mathcal{L},-1}\right)_{*} \operatorname{IC}\left(\operatorname{Hess}_{\mathcal{L},-1}, \hat{\mathcal{L}}_{-1}\right)\right)$ as a direct summand.

Assume from now on that $\pi_{\mathcal{L},-1}: \operatorname{Hess}_{\mathcal{L},-1} \rightarrow \mathfrak{g}_{1}$ is surjective. Then the sheaf $\left(\pi_{\mathcal{L},-1}\right)_{*} \operatorname{IC}\left(\operatorname{Hess}_{\mathcal{L},-1}, \hat{\mathcal{L}}_{-1}\right)$ is smooth over $\mathfrak{g}_{1}^{r s}$. One sees this as follows. According to the first 
statement of $(\underline{6.1})$ the characteristic variety of $\left(\pi_{\mathcal{L},-1}\right)_{*} \operatorname{IC}\left(\operatorname{Hess}_{\mathcal{L},-1}, \hat{\mathcal{L}}_{-1}\right)$ coincides with that of $\left(\pi_{\mathcal{L}, 1}\right)_{*} \operatorname{IC}\left(\operatorname{Hess}_{\mathcal{L}, 1}, \hat{\mathcal{L}}_{1}\right)$ as they are Fourier transforms of each other. But $\left(\pi_{\mathcal{L}, 1}\right)_{*} \operatorname{IC}\left(\operatorname{Hess}_{\mathcal{L}, 1}, \hat{\mathcal{L}}_{1}\right)$ is $K$-equivariant and supported on the nilpotent cone. A straightforward calculation then shows the smoothness of $\left(\pi_{\mathcal{L},-1}\right)_{*} \operatorname{IC}\left(\operatorname{Hess}_{\mathcal{L},-1}, \hat{\mathcal{L}}_{-1}\right)$ on $\mathfrak{g}_{1}^{r s}$. Thus, by the decomposition theorem, we conclude that:

$$
\left.\left(\pi_{\mathcal{L},-1}\right)_{*} \mathrm{IC}\left(\operatorname{Hess}_{\mathcal{L},-1}, \hat{\mathcal{L}}_{-1}\right)\right) \text { is a direct sum of shifts of irreducible local systems . }
$$

In addition, the $\mathrm{IC}\left(\operatorname{Hess}_{\mathcal{L},-1}, \hat{\mathcal{L}}_{-1}\right)$ and hence $\left(\pi_{\mathcal{L},-1}\right)_{*} \mathrm{IC}\left(\operatorname{Hess}_{\mathcal{L},-1}, \hat{\mathcal{L}}_{-1}\right)$ has a canonical structure as a Hodge module and thus the direct summands are IC-extensions of irreducible variations of pure Hodge structure, see, $[\mathrm{S}]$.

We fix a generic $s \in \mathfrak{g}_{1}^{r s}$ and then

$$
H^{*}\left(\left(\pi_{\mathcal{L},-1}\right)_{*} \operatorname{IC}\left(\operatorname{Hess}_{\mathcal{L},-1}, \hat{\mathcal{L}}_{-1}\right)\right)_{s}=\operatorname{IH}^{*}\left(\operatorname{Hess}_{\mathcal{L},-1, s}, \hat{\mathcal{L}}_{-1}\right) .
$$

Thus we obtain an action of the fundamental group $\pi_{1}^{K}\left(\mathfrak{g}_{1}^{r s}, s\right)$ on $\operatorname{IH}^{*}\left(\operatorname{Hess}_{\mathcal{L},-1, s}, \hat{\mathcal{L}}_{-1}\right)$ and by the discussion above this action breaks into a direct sum of irreducible representations which are also variations of Hodge structure.

The component group $\pi_{0}\left(Z_{K}(s)\right) \cong I_{N}$ acts on $\operatorname{IH}^{*}\left(\operatorname{Hess}_{\mathcal{L},-1, s}, \hat{\mathcal{L}}_{-1}\right)$ and we write

$$
\operatorname{IH}^{*}\left(\operatorname{Hess}_{\mathcal{L},-1, s}, \hat{\mathcal{L}}_{-1}\right)=\bigoplus_{\chi \in I_{N}^{\vee}} \operatorname{IH}^{*}\left(\operatorname{Hess}_{\mathcal{L},-1, s}, \hat{\mathcal{L}}_{-1}\right)_{\chi}
$$

for the corresponding eigenspace decomposition.

Definition 6.1. The stable part $\operatorname{IH}^{*}\left(\operatorname{Hess}_{\mathcal{L},-1, s}, \hat{\mathcal{L}}_{-1}\right)_{\mathrm{st}}$ of $\operatorname{IH}^{*}\left(\operatorname{Hess}_{\mathcal{L},-1, s}, \hat{\mathcal{L}}_{-1}\right)$ is the direct summand $\operatorname{IH}^{*}\left(\operatorname{Hess}_{\mathcal{L},-1, s}, \hat{\mathcal{L}}_{-1}\right)_{\chi_{\text {triv }}}$ where $\chi_{\text {triv }} \in I_{N}^{\vee}$ is the trivial character.

Observe that $\operatorname{IH}^{*}\left(\operatorname{Hess}_{\mathcal{L},-1, s}, \hat{\mathcal{L}}_{-1}\right)_{\text {st }}$ is stable under the monodromy action of $\pi_{1}^{K}\left(\mathfrak{g}_{1}, s\right)$. Moreover, the action factors through the braid group $B_{N}$ via the quotient map $\pi_{1}^{K}\left(\mathfrak{g}_{1}, s\right) \rightarrow$ $B_{N}$.

For every irreducible representation $D_{\mu}$ of $\mathcal{H}_{N,-1}$, let $V_{\mu}$ be the local system on $\mathfrak{g}_{1}^{\text {rs }}$ associated to $D_{\mu}$. By Theorem 4.1, there exists a unique nilpotent pair $\left(\mathcal{O}_{\mu}, \mathcal{L}_{\mu}\right)$ such that $\mathfrak{F}\left(\mathrm{IC}\left(\overline{\mathcal{O}}_{\mu}, \mathcal{L}_{\mu}\right)\right) \cong \mathrm{IC}\left(\mathfrak{g}_{1}, V_{\mu}\right)$.

Theorem 6.1. Let $D_{\mu}$ be an irreducible representation of $\mathcal{H}_{N,-1}$ and let $\left(\mathcal{O}_{\mu}, \mathcal{L}_{\mu}\right)$ be the associated nilpotent pair as above. We have

(1) The map $\pi_{\mathcal{L}_{\mu},-1}$ is onto, the action of the braid group $B_{N}$ on $\operatorname{IH}^{*}\left(\operatorname{Hess}_{\mathcal{L}_{\mu},-1, s}, \hat{\mathcal{L}}_{\mu,-1}\right)_{\mathrm{st}}$ factors through the Hecke algebra $\mathcal{H}_{N,-1}$ and $\mathrm{IH}^{*}\left(\operatorname{Hess}_{\mathcal{L}_{\mu},-1, s}, \hat{\mathcal{L}}_{\mu,-1}\right)_{\text {st }}$ is a direct sum of irreducible representations of $\mathcal{H}_{N,-1}$.

(2) $D_{\mu}$ appears in $\mathrm{IH}^{*}\left(\operatorname{Hess}_{\mathcal{L}_{\mu},-1, s}, \hat{\mathcal{L}}_{\mu,-1}\right)_{\mathrm{st}}$ with non-zero multiplicity.

Proof. Since for every irreducible subrepresentation $W$ of $\operatorname{IH}^{*}\left(\operatorname{Hess}_{\mathcal{L}_{\mu},-1, s}, \hat{\mathcal{L}}_{\mu,-1}\right)_{\mathrm{st}}$ the corresponding Fourier transform $\mathfrak{F}\left(\operatorname{IC}\left(\mathfrak{g}_{1}, \mathcal{W}\right)\right)$ is supported on the nilpotent cone (here $\mathcal{W}$ is the 
local system on $\mathfrak{g}_{1}^{r s}$ associated to $W$ ), the same argument as in the proof of Theorem 5.1 implies part (1). Part (2) follows from (6.1), (6.2), and (6.4).

\section{Conjecture on more Precise matching}

In Theorem 4.1 we show that the Fourier transform establishes a bijection between two sets of intersection cohomology sheaves. In this section we formulate a conjecture which refines the bijection in Theorem 4.1. We also relate the conjecture to our earlier conjectures in CVX2]. Our conjecture is not strong enough to produce and exact matching. The exact description of the bijection is crucial for applications, for example, computing cohomologies of Hessenberg varieties as explained in Section 5 .

We begin with associating to each nilpotent orbit $\mathcal{O}_{\lambda}$ (resp. $\mathcal{O}_{\lambda}^{\omega}, \omega=$ I, II) a subset $\Sigma_{\lambda} \subset \Sigma_{N}$ (resp. $\Sigma_{\lambda}^{\omega} \subset \Sigma_{N}$ ), if $\lambda \in \mathcal{P}(N)$ has at least one odd part (resp. has only even parts).

Let $\lambda$ be a partition of $N$ and let $\lambda^{\prime}$ be the transpose partition of $\lambda$. Suppose that

$$
\lambda^{\prime}=\left(\lambda_{1}^{\prime}\right)^{2 m_{1}} \cdots\left(\lambda_{l}^{\prime}\right)^{2 m_{l}}\left(\lambda_{l+1}^{\prime}\right)^{2 m_{l+1}-1} \cdots\left(\lambda_{k}^{\prime}\right)^{2 m_{k}-1},
$$

where $m_{i} \geq 1, i=1, \ldots, k$. Here and in what follows we write the parts in a partition in the order which is most convenient for us. In parcticular, in (7.1) we place the parts with even multiplicity before the parts with odd multiplicity.

Let $\delta_{i} \in\{0,1\}$ for $i \in[1, l]$ and let

$$
\begin{gathered}
\nu\left(\delta_{1}, \ldots, \delta_{l}\right)=\left(\lambda_{1}^{\prime}\right)^{m_{1}-\delta_{1}} \cdots\left(\lambda_{l}^{\prime}\right)^{m_{l}-\delta_{l}}\left(\lambda_{l+1}^{\prime}\right)^{m_{l+1}-1} \cdots\left(\lambda_{k}^{\prime}\right)^{m_{k}-1}, \\
\mu\left(\delta_{1}, \ldots, \delta_{l}\right)=\left(\lambda_{1}^{\prime}\right)^{2 \delta_{1}} \cdots\left(\lambda_{l}^{\prime}\right)^{2 \delta_{l}}\left(\lambda_{l+1}^{\prime}\right) \cdots\left(\lambda_{k}^{\prime}\right) .
\end{gathered}
$$

Note that $2\left|\nu\left(\delta_{1}, \ldots, \delta_{l}\right)\right|+\left|\mu\left(\delta_{1}, \ldots, \delta_{l}\right)\right|=N$. Let

$$
J \subset J_{0}:=\{l+1, \ldots, k\} \text { such that } \sum_{j \in J} \lambda_{j}^{\prime}<\sum_{j \in J_{0}-J} \lambda_{j}^{\prime} .
$$

We define

$$
\begin{gathered}
\mu^{1}\left(\delta_{1}, \ldots, \delta_{l} ; J\right)=\left(\lambda_{1}^{\prime}\right)^{\delta_{1}} \cdots\left(\lambda_{l}^{\prime}\right)^{\delta_{l}}\left(\lambda_{j_{1}}^{\prime}\right) \cdots\left(\lambda_{j_{s}}^{\prime}\right), J=\left\{j_{1}, \ldots, j_{s}\right\} . \\
\mu^{2}\left(\delta_{1}, \ldots, \delta_{l} ; J\right)=\left(\lambda_{1}^{\prime}\right)^{\delta_{1}} \cdots\left(\lambda_{l}^{\prime}\right)^{\delta_{l}}\left(\lambda_{i_{1}}^{\prime}\right) \cdots\left(\lambda_{i_{k-l-s}}^{\prime}\right), J_{0}-J=\left\{i_{1}, \ldots, i_{k-l-s}\right\} .
\end{gathered}
$$

Note that $\lambda_{l+1}^{\prime}=0$ if and only if all parts of $\lambda$ are even. In this case, $J_{0}=\emptyset=J$ and $\mu^{1}\left(\delta_{1}, \ldots, \delta_{l} ; J\right)=\mu^{2}\left(\delta_{1}, \ldots, \delta_{l} ; J\right)$ and we write $\mu\left(\delta_{1}, \ldots, \delta_{l}\right)=\mu^{i}\left(\delta_{1}, \ldots, \delta_{l} ; J\right), i=1,2$.

If $\lambda$ has at least one odd part, then let

$$
\begin{gathered}
\Sigma_{\lambda}:=\left\{\left(\nu\left(\delta_{1}, \ldots, \delta_{l}\right) ; \mu^{1}\left(\delta_{1}, \ldots, \delta_{l} ; J\right), \mu^{2}\left(\delta_{1}, \ldots, \delta_{l} ; J\right)\right) \mid \delta_{i} \in\{0,1\}, i=1, \ldots, l,\right. \\
\left.J \subset\{l+1, \ldots, k\}, \text { such that } \sum_{j \in J} \lambda_{j}^{\prime}<\sum_{j \in J_{0}-J} \lambda_{j}^{\prime}\right\} .
\end{gathered}
$$


If all parts of $\lambda$ are even (in which case $\lambda_{l+1}^{\prime}=0$ ), then let

$$
\Sigma_{\lambda}^{\omega}=\left\{\left(\nu\left(\delta_{1}, \ldots, \delta_{l}\right) ; \mu\left(\delta_{1}, \ldots, \delta_{l}\right), \mu\left(\delta_{1}, \ldots, \delta_{l}\right)\right)^{\omega} \mid \delta_{i} \in\{0,1\}, i=1, \ldots, l\right\}, \omega=\mathrm{I}, \mathrm{II} .
$$

We have $\left|\Sigma_{\lambda}\right|=2^{k-1}$ (resp. $\left|\Sigma_{\lambda}^{\omega}\right|=2^{l}$ ), which equals the number of non-isomorphic irreducible $K$-equvariant local systems on $\mathcal{O}_{\lambda}$ (resp. $\left.\mathcal{O}_{\lambda}^{\omega}\right)$.

Conjecture 7.1. Let $\lambda$ be a partition of $N$.

(1) If $\lambda$ has at least one odd part, then the Fourier transform $\mathfrak{F}$ induces the following bijection

$\mathfrak{F}:\left\{\operatorname{IC}\left(\mathcal{O}_{\lambda}, \mathcal{E}\right) \mid \mathcal{E}\right.$ irreducible $K$-equivariant local system on $\mathcal{O}_{\lambda}$ (up to isomorphism) $\}$

$$
\stackrel{\sim}{\rightarrow}\left\{\mathrm{IC}\left(\mathfrak{g}_{1}^{|\nu|}, \mathcal{T}\left(\nu ; \mu^{1}, \mu^{2}\right)\right) \mid\left(\nu ; \mu^{1}, \mu^{2}\right) \in \Sigma_{\lambda}\right\} .
$$

Moreover,

$$
\mathfrak{F}\left(\operatorname{IC}\left(\mathcal{O}_{\lambda}, \mathbb{C}\right)\right)=\operatorname{IC}\left(\mathfrak{g}_{1}^{\left|\nu_{0}\right|}, \mathfrak{T}\left(\nu_{0} ; \mu_{0}^{1}, \mu_{0}^{2}\right)\right)
$$

where $\left(\nu_{0} ; \mu_{0}^{1}, \mu_{0}^{2}\right) \in \Sigma_{\lambda}$ is the unique triple such that $\left|\nu_{0}\right|=\max \left\{|\nu|,\left(\nu, \mu^{1}, \mu^{2}\right) \in \Sigma_{\lambda}\right\}$ and the parts of $\mu_{0}^{1}$ and the parts of $\mu_{0}^{2}$ have the opposite parity (in particular, all parts of $\mu_{0}^{i}$ have the same parity).

(2) If all parts of $\lambda$ are even, then the Fourier transform induces the following bijection $\mathfrak{F}:\left\{\operatorname{IC}\left(\mathcal{O}_{\lambda}^{\omega}, \mathcal{E}\right) \mid \omega=\mathrm{I}, \mathrm{II}, \mathcal{E}\right.$ irreducible K-equivariant local system on $\mathcal{O}_{\lambda}^{\omega}$ (up to isom) $\}$

$$
\begin{gathered}
\stackrel{\sim}{\rightarrow}\left\{\mathrm{IC}\left(\mathfrak{g}_{1}^{|\nu|}, \mathcal{T}(\nu ; \mu, \mu)^{\omega}\right) \mid \omega=\mathrm{I}, \mathrm{II}, \quad(\nu ; \mu, \mu)^{\omega} \in \Sigma_{\lambda}^{\omega}, \mu \neq \emptyset\right\} \\
\cup\left\{\mathrm{IC}\left(\mathfrak{g}_{1}^{n, \omega}, \mathcal{T}(\nu ; \emptyset, \emptyset)\right) \mid \omega=\mathrm{I}, \mathrm{II}, \quad(\nu ; \emptyset, \emptyset) \in \Sigma_{\lambda}^{\omega}\right\} .
\end{gathered}
$$

Moreover,

$$
\mathfrak{F}\left(\operatorname{IC}\left(\mathcal{O}_{\lambda}^{\omega}, \mathbb{C}\right)\right)=\operatorname{IC}\left(\mathfrak{g}_{1}^{n, \omega}, \mathcal{T}\left(\nu_{0} ; \emptyset, \emptyset\right)\right)
$$

where $\left|\nu_{0}\right|=n$ and $\left(\nu_{0} ; \emptyset, \emptyset\right) \in \Sigma_{\lambda}$.

Note that $\mathfrak{F}\left(\operatorname{IC}\left(\mathcal{O}_{\lambda}, \mathcal{E}\right)\right)$ has full support if and only if $\nu\left(\delta_{1}, \ldots, \delta_{l}\right)=\emptyset$. Thus we see that the Conjecture is compatible with Corollary 4.9.

Let us relate the conjecture above to our previous conjectures in CVX2]. In CVX2] we constructed local systems $E_{i, j}^{2 n+1}$ and $\widetilde{E}_{i, j}^{2 n+1}$ on $\mathfrak{g}_{1}^{r s}$. In terms of the parametrization introduces in this paper, we have

$$
\begin{gathered}
E_{i, j}^{2 n+1}=\mathcal{T}(\emptyset ;(2 i-j, j),(2 n+1-2 i)) \\
\widetilde{E}_{i, j}^{2 n+1}=\mathcal{T}(\emptyset ;(2 i-1-j, j),(2 n+2-2 i)) .
\end{gathered}
$$

Thus we see that Conjecture 7.1 applied to $E_{i, j}^{2 n+1}$ agrees with Conjectures 6.1 and 6.3 in CVX2]. Applied to $\widetilde{E}_{i, j}^{2 n+1}$, Conjecture 7.1 implies that the supports of $\mathfrak{F}\left(\operatorname{IC}\left(\mathfrak{g}_{1}, \widetilde{E}_{i, j}^{2 n+1}\right)\right)$ are 
as follows:

$$
\begin{gathered}
\mathcal{O}_{3^{j} 2^{2 i-2 j-1} 1^{2 n+3-4 i+j}} \text { if } 4 i-j \leq 2 n+3 \\
\mathcal{O}_{3^{j} 2^{2 n+2-2 i-j} 1^{4 i-j-2 n-3}} \text { if } 2 i+j \leq 2 n+2 \text { and } 4 i-j \geq 2 n+3 \\
\mathcal{O}_{3^{2 n-2 i+2} 2^{2 i+j-2 n-2} 1^{2 i-2 j-1}} \text { if } 2 i+j \geq 2 n+2 .
\end{gathered}
$$

Note that the above orbits are all of even dimensional and each of the even-dimensional orbits appears twice there.

\section{REFERENCES}

[A] A'Campo, Norbert. Tresses, monodromie et le groupe symplectique, Comment. Math. Helv. 54 (1979), no. 2, 318-327.

[BH] M. Brion and A. G. Helminck, On orbit closures of symmetric subgroups in flag varieties, Canad. J. Math. 52 (2000), no. 2, 265-292.

[CVX1] T.H. Chen, K. Vilonen, and T. Xue. Springer correspondence for symmetric spaces. Arxiv.1510.05986.

[CVX2] T.H. Chen, K. Vilonen, and T. Xue. Hessenberg varieties, intersections of quadrics, and the Springer correspondence. arXiv:1511.00617.

[CVX3] T.H. Chen, K. Vilonen, and T. Xue. Cohomology of Hessenberg varieties and Springer correspondence . Arxiv.1510.05986.

[DJ] R. Dipper and G. James, Representations of Hecke algebras of general linear groups, Proc. London Math. Soc. (3) 52 (1986), no. 1, 20-52.

[GS] W. M. Y. Goh and E. Schmutz, The number of distinct part sizes in a random integer partition, J. Combin. Theory Ser. A 69 (1995), no. 1, 149-158.

[GKM] M. Goresky, R. Kottwitz and R. MacPherson, Purity of equivalued affine Springer fibers, Represent. Theory 10 (2006), 130-146 (electronic).

[G1] M. Grinberg, On the specialization to the asymptotic cone, J. Algebraic Geom. 10 (2001), no. 1, $1-17$.

[G2] M. Grinberg, A generalization of Springer theory using nearby cycles, Represent. Theory 2 (1998), 410-431 (electronic).

[Gr] I. Grojnowski, Character sheaves on symmetric spaces. Thesis (Ph.D.)Massachusetts Institute of Technology (1992).

[H] A. Henderson, Fourier transform, parabolic induction, and nilpotent orbits, Transform. Groups 6 (2001), no. 4, 353-370.

[KR] B. Kostant and S. Rallis, Orbits and representations associated with symmetric spaces, Amer. J. Math. 93 (1971), 753-809.

[L] G. Lusztig, Study of antiorbital complexes, in Representation theory and mathematical physics, 259-287, Contemp. Math., 557, Amer. Math. Soc., Providence, RI.

[L1] G. Lusztig, Study of perverse sheaves arising from graded Lie algebras, Advanced in Mathematics 112 (1995), 147-217.

[LY] G. Lusztig and Z. Yun, Z/m-graded Lie algebras and perverse sheaves, I, arXiv:1602.05244.

[LS] G. Lusztig and N. Spaltenstein, Induced unipotent classes, J. London Math. Soc. (2) 19 (1979), no. $1,41-52$.

[S] M. Saito, Modules de Hodge polarisables, Publ. Res. Inst. Math. Sci. 24 (1988), no. 6, 849-995. 
Department of Mathematics, University of Chicago, Chicago, 60637, USA

E-mail address: tsaohsien@gmail.com

School of Mathematics and Statistics, University of Melbourne, ViC 3010, Australia, and Department of Mathematics and Statistics, University of Helsinki, Helsinki, 00014, FINLAND

E-mail address: kari.vilonen@unimelb.edu.au

School of Mathematics and Statistics, University of Melbourne, ViC 3010, Australia, and Department of Mathematics and Statistics, University of Helsinki, Helsinki, 00014, FINLAND

E-mail address: ting.xue@unimelb.edu.au 